\title{
Radiocarbon
}

\author{
1978
}

\section{LA JOLLA MEASUREMENTS OF RADIOCARBON IN TREE-RING DATED WOOD}

\author{
H E SUESS
}

Mt Soledad Radiocarbon Laboratory, Department of Chemistry, University of California, San Diego, La Jolla, California 92093

In 1969, at the Nobel Symposium on "Radiocarbon Variations and Absolute Chronology" in Uppsala, a curve was presented that illustrated the functional dependence of measured conventional radiocarbon dates on true historical ages of wood samples (Suess, 1971). The curve was derived from the results of La Jolla measurements of radiocarbon in bristlecone pine wood dendrochronologically dated by and obtained from Professor C W Ferguson of the University of Arizona (Ferguson, 1968). The curve was intended to be adequate for deriving calibrated radiocarbon dates and also for allowing fairly reliable estimates of the accuracy of the absolute dates obtained in this manner. The basis for the validity of this calibration is the well known fact that, for all practical purposes, wood samples that had grown at the same time show the same radiocarbon content. However, the reverse is not always true: Wood samples showing the same radiocarbon content do not necessarily have the same age because of the windings and steps of the curve (Stuiver and Suess, 1966).

An accurate knowledge of the secular variations of radiocarbon is desirable not only for calibration purposes but also for understanding the geophysical and geochemical factors that cause these variations. The curve published in 1971 illustrated appropriately the character of the variations but was not sufficiently complete to show quantitatively the fine structure features and details that were undoubtedly present. Measurements by other laboratories confirmed the general trend of the curve but have not contributed to our knowledge of the more rapid fluctuations on a time scale of some 100 ys, the so-called "wiggles" of the calibration curve.

Since 1969 our measurements on tree-ring dated wood have been carried out primarily for the purpose of obtaining more detailed information on secular radiocarbon variations. For many years the number of measurements that could be carried out was limited by the number of samples that Profesor Ferguson made available. Unfortunately, these samples often did not come from a time range that appeared particularly interesting. Since 1972, however, long "floating" tree-ring sequences from logs of oak trees in riverbeds of central Europe have been compiled by Becker (1978). Results of measurements from these sequences and their comparisons with bristlecone pine results were presented at the Conference on Dendrochronology at Mainz in 1974 (Suess and Becker, 1977). It was shown that 
the " $\delta{ }^{14} \mathrm{C}$ wiggles" were synchronous in the wood of European oak and of California bristlecone pine and that they had the same amplitudes within limits of errors of the measurements. About 200 numerical values of $\delta{ }^{14} \mathrm{C}$ were published at that time. Samples from these oak trees, obtained from Becker, have also made it possible to carry out a sufficient number of measurements for the 4th millenium BC, so that a consistent picture of the variations during this time interval could be established. A graphic presentation of these results was published at the Ninth International Radiocarbon Conference in 1976 (Suess, 1978); their numerical values are included in the tables below. Some values deviate slightly from those given previously because of minor adjustments in counter calibrations.

It was intended to postpone publication of the individual numerical values of these and of the additional results until a statistical analysis of the data, as well as "computer-matching" of floating data with dendrochronologically derived absolute data, and also Fourier analyses could be carried out. Unfortunately, National Science Foundation funding for this purpose was denied, and it now appears that it will take another year or two before the reduction and interpretation of the experimentally obtained raw data will be completed. Therefore, following the requests of many colleagues, the conventional "Libby" ages obtained for wood samples, dendrochronologically dated or belonging to "floating" tree-ring series, are now listed here, in sequence of their ages. The results of the analyses of the data, and their discussion, in particular with respect to their geophysical significance, will be published later.

The results listed in the following tables are published here primarily to help to derive calibrated radiocarbon dates and to estimate their accuracy. At the time the Uppsala curve was published, it was not known that the two Öeschger-Houtermans counters (denoted here B and M respectively) used (Linick, 1976) showed a memory, even after three or four hours of pumping. The errors caused by this memory, in general, did not exceed expected statistical counting errors of the individual measurements but could be recognized by combining a number of results. Many standard and background measurements appeared affected by the ${ }^{14} \mathrm{C}$-content of the preceding samples, so that all the results on which the curve was based had to be recalculated with revised values. Also, results that were obtained in the run immediately following the counting of a gas with considerably different radiocarbon content, such as a background or standard gas, were routinely omitted. These revised values deviate significantly only for limited time periods from those from which the Uppsala curve was derived. For optimally accurate conversion of conventional radiocarbon dates it is best simply to compare the numerical value of the conventional radiocarbon date of a given sample with those listed in appendix 1.

Column 1 of appendixes 1 and 2: Measurements were carried out approximately in sequence of their LJ-number. Before 1965 they were 
assigned LJ-numbers smaller than LJ-1150. These results were numerically published before (Suess, 1965) and are not included here.

Except for the results that were excluded for the reasons given above, all data obtained in La Jolla before Sept 1977 for radiocarbon in treerings are listed in the two appendices. No further attempts were made to screen the results, although, as can easily be seen, some of them are undoubtedly in error. For example, for several measurements the results from the two sets of counting equipment ( $\mathrm{B}$ and $\mathrm{M}$ ) differ by considerably more than the expected statistical variation, indicating that one of the two sets was out of order. It will certainly be possible to find and exclude questionable results on the basis of well defined criteria after an analysis of the raw data is made. For further remarks on errors see below.

Until 1969 the La Jolla Radiocarbon Laboratory was located in the basement of Ritter Hall, Scripps Institute of Oceanography. Measurements carried out there have LJ-numbers smaller than LJ 1930. In 1970 the laboratory moved to a new building on Mt Soledad, interrupting operations for about 6 months. During the following 2 years determinations were primarily carried out of radiocarbon in wood less than 100 ys old (Cain and Suess, 1976). These results were not included. In 1972, B Becker of the University of Stuttgart-Hohenheim made available the first samples from his floating European oak chronology. The LJ-numbers of the measurements listed in the two tables correspond approximately to the following years of operations:

$\begin{array}{rc}1965 \text { to } 1969: & \text { LJ } 1150-\text { LP } 1930 \\ 1970 \text { to 1973: } & \text { LJ 2150- LP } 2890 \\ 1974: & \text { LJ } 2900-\text { LJ-3175 } \\ 1975: & \text { LJ } 3180-\text { LJ } 3465 \\ 1976: & \text { LJ } 3465-\text { LJ } 3800 \\ \text { to 8-31-1977: } & >\text { LJ } 3800\end{array}$

Column 2 of appendix 1: TRL numbers denote bristlecone pine samples and identify the respective tree from which the sample came (Ferguson, 1968). For dendrochronologically dated European samples (available back to $800 \mathrm{BC}$, the time for which an absolute tree ring chronology was established in Europe) the name of the submitter is listed (for provenience of these samples see Becker (1978) and Holstein [to be published]).

In appendix 2 the name of the floating tree-ring series and the name of the submitter (unless submitted by Becker) is listed in this column.

Column 3: Appendix 1 gives the midpoints of the years of growth of the sequence of rings used for the measurements. Negative numbers denote $\mathrm{BC}$ dates, except that, as Ferguson is anxious to point out, these numbers differ from the historical BC dates by 1 year because historians do not use a year zero in their time scale, whereas dendrochronologists and geophysicists do. In appendix 2 this column gives the midpoints of the respective ring numbers of the rings used for the measurements.

Column 4: The number of rings used for each measurement is listed here. With a few exceptions Ferguson makes available samples of ten rings 
of bristlecone pine wood. From European oak trees, samples consisting of one single ring can frequently be obtained.

Column 5 gives the measured $\delta{ }^{13} \mathrm{C}$ as conventionally defined. These values however are not necessarily those for the $\delta{ }^{13} \mathrm{C}$ in the original wood sample but rather for the gas used for counting. Until approximately 1967 , this counting gas, acetylene, was prepared via strontium carbide. It can be seen from the $\delta{ }^{13} \mathrm{C}$ values that this method leads to a much greater isotope fractionation than the method using lithium carbide which is now employed. Values in parentheses are estimates used for the calculations of the ages. Until 1970 the $\delta{ }^{13} \mathrm{C}$ measurements were made in the laboratories of Harmon Craig and Samuel Epstein. Since then the old Chicago mass spectrometer of the laboratory of $\mathrm{H} \mathrm{C}$ Urey, now in the hands of $\mathrm{K}$ Marti, is being used.

Column 6 lists the results expressed in conventional (Libby) radiocarbon years. With a few exceptions they were obtained by counting in succession for about $40 \mathrm{hrs}$ in each of two Öeschger Houtermans counters. The characteristics of the two counters were published previously (Linick, 1977). Approximately 20 grams of wood was available for each measurement. The wood was treated with acetone, azide, and base as described previously (Cain and Suess, 1976; Linick, 1977).

Column 7 lists the statistical one sigma standard counting errors of the results. These errors include the statistical uncertainty of backgrounds and standards but not the uncertainties in the ${ }^{14} \mathrm{C}$-contents of the used standards. Until about 1973 late 19th century wood specimens were used as standards. Comparison measurements with the NBS oxalic acid standard were made only occasionally. It appears now, that the ${ }^{14} \mathrm{C}$ content in these wood samples may have varied by some 30 per mil, but no accurate figure can be given as such variations are well within the accuracy of the comparison measurements with the NBS oxalic acid, and additional wood to repeat these measurements is no longer available. During the more recent years both wood as well as NBS oxalic acid were used as standards. The conventional radiocarbon ages of these samples were obtained relative to a radiocarbon content of $0.944 \pm 0.002$ times that of the NBS oxalic acid. No attempts were made to correct for the differences in the used standards, although minor corrections by "computer matching" of data sequences obtained earlier with those obtained with optimally defined standards may be possible and will be attempted.

For several reasons a decrease in the listed statistical errors by increasing counting times and/or counter volumns is of no advantage and uneconomical. With the exception of some rare cases of malfunctioning of electronics, the main additional errors appear to be due to carbon impurities in the samples. In order to avoid these completely, the cellulose would have to be separated and purified, but neither sufficient wood quantities nor necessary manpower is available to carry out such laborious additional work. 
Column 8 lists the difference in years of the results obtained for each sample from the two sets of counting equipment $\mathrm{M}$ and $\mathbf{B}$. For some samples only one valid measurement was obtained, and in these cases the symbol for the set in which the result was obtained is shown instead of the difference.

As can be seen from the tables, the number of measurements for a given time interval varies greatly over the total period for which measurements have been carried out which is from $1300 \mathrm{AD}$ to around $6000 \mathrm{BC}$. This is in part a consequence of the limited availability of samples and in part due to the fact that some periods of time appeared particularly interesting. Nevertheless, from the samples so far measured it can be seen already that the carbon-14 in the atmosphere did not fluctuate randomly but followed certain patterns so that one might be tempted to believe in the existence of cycles or at least in the significance of certain "magic numbers" of years. It can be expected that further measurements, which will be carried out during the next year or two, will complete the picture for the whole period of time for which tree-ring dated wood is available and will then make it possible to decide whether or not the observed patterns have a true physical meaning. It will then be time to look for correlations with other geophysical and geochemical parameters and to speculate about the explanations and interpretations of the findings.

\section{ACKNOWLEDGMENTS}

The results reported here represent only a fraction of the total number of measurements that are being carried out by the laboratory. Thanks to the unusual enthusiasm, efficiency, and dedication of the staff, in particular of Ms Carol Hutto and Dr Timothy Linick, the laboratory has for more than 6 years operated practically uninterruptedly on a 24 hour a day, 7 day a week basis. Besides taking care of all the technical operations for the radiocarbon determinations, Dr Linick is carrying out computerized data reductions and, together with Ms Hutto, mass spectrometric carbon-13 determinations. In this connection thanks are due also to Drs $\mathrm{K}$ Marti and G Lugmair for assistance and advice in the mass spectrometer operations.

As always it should be emphasized that this work is based entirely on the tree-ring sequence established for the past 8000 yrs by C W Ferguson. The dendrochronologically dated wood samples came from Dr. Ferguson with the exception of some samples from the European chronology that extends back to $800 \mathrm{BC}$. Almost all the samples from the floating chronologies were obtained from B Becker of the University of Stuttgart-Hohenheim. Dr E Hollstein, Landesmuseum Trier, supplied samples from the fifth and sixth century BC.

Operation of the La Jolla Radiocarbon Laboratory is financed by the National Science Foundation, Division of Earth Sciences, Geochemistry Program through Grant EAR76-22623, and predecessor grants. 
APPENDIX 1

Conventional radiocarbon ages of dendrochronologically-dated wood (TRL samples supplied by C W Ferguson; Holstein samples by E Holstein, Trier, W Germany, to be pub; all others by B Becker, 1978.)

\begin{tabular}{|c|c|c|c|c|c|c|c|}
\hline 1. & 2. & 3. & 4. & 5. & 6. & 7. & 8. \\
\hline LJ-3314 & TRL $70-46$ & -6045 & 10 & -21.3 & 7095 & 51 & 73 \\
\hline LJ-3306 & TRL 70-46 & -6025 & 10 & -20.4 & 7116 & 51 & -220 \\
\hline LJ -3312 & TRL 70-46 & -6015 & 10 & -20.1 & 7149 & 51 & 78 \\
\hline LJ-2874 & TRL 70-46 & -6015 & 10 & -20.9 & 7027 & 57 & 154 \\
\hline LJ -2875 & TRL $70-46$ & -5995 & 10 & -21.5 & 7119 & 57 & 87 \\
\hline LJ-3311 & TRL $70-46$ & -5995 & 10 & -21.1 & 7187 & 52 & 79 \\
\hline LJ'-3310 & TRL $70-46$ & -5985 & 10 & -20.7 & 7117 & 51 & 125 \\
\hline LJ-3313 & TRL $70-46$ & -5975 & 10 & -20.0 & 7046 & 50 & -25 \\
\hline LJ -2877 & TRL $70-46$ & -5975 & 10 & -21.3 & 7087 & 57 & 69 \\
\hline LJ -3308 & TRL $70-46$ & -5965 & 10 & -21.1 & 6949 & 51 & -247 \\
\hline LJ -2196 & TRL $70-20$ & -5402 & 10 & -21.1 & 6352 & 60 & -17 \\
\hline LJ-2206 & TRL $70-20$ & -5362 & 10 & -21.6 & 6248 & 58 & -6 \\
\hline LJ-1921 & TRL 67-35 & -5290 & 14 & -21.9 & 6111 & 87 & -68 \\
\hline LJ -2207 & TRL $70-20$ & -5282 & 10 & -21.4 & 6117 & 59 & 9 \\
\hline LJ -2198 & TRL $70-20$ & -5263 & 10 & -21.5 & 6311 & 59 & -67 \\
\hline LJ J-2204 & TRL $70-20$ & -5243 & 10 & -21.2 & 6143 & 60 & -39 \\
\hline LJ -2194 & TRL $70-20$ & -5223 & 10 & -21.2 & 6224 & 62 & 28 \\
\hline LJ -2197 & TRL $70-20$ & -5203 & 10 & -21.2 & 6126 & 60 & 38 \\
\hline LJ-2435 & TRL 70-20 & -5182 & 10 & -21.8 & 6163 & 55 & 132 \\
\hline LJ-2439 & TRL $70-20$ & -5162 & 10 & -22.2 & 6188 & 55 & -56 \\
\hline LJ -2395 & TRL $70-20$ & -5143 & 10 & -20.4 & 6049 & 61 & 60 \\
\hline LJ-1538 & TRL 63-92E & -5083 & 54 & $(-22.0)$ & 6082 & 68 & 20 \\
\hline LJ-1492 & TRL 63-92E & -5075 & 10 & -21.8 & 6139 & 63 & 29 \\
\hline LJ-1713 & TRL 63-92E & -5055 & 10 & -21.9 & 6031 & 75 & B \\
\hline $\mathrm{LJ}-1712$ & TRL 63-92E & -5035 & 10 & -21.4 & 6151 & 96 & $\mathbf{M}$ \\
\hline LJ-1734 & TRL $63-92 \mathbf{E}$ & -4965 & 10 & $(-22.0)$ & 6009 & 59 & -85 \\
\hline LJ-1732 & TRL 63-92E & -4945 & 10 & $(-22.0)$ & 5958 & 58 & -73 \\
\hline LJ J-1739 & TRL 63-92E & -4895 & 10 & -22.5 & 5921 & 58 & 14 \\
\hline L.J-1740 & TRL 63-92E & -4875 & 10 & -22.0 & 5916 & 58 & 12 \\
\hline LJ-1539 & TRL 63-92E & -4795 & 10 & -21.4 & 5969 & 66 & 5 \\
\hline LJ -2164 & TRL $63-92 \mathrm{E}$ & -4753 & 16 & --22.2 & 5784 & 57 & -5 \\
\hline LJ -2179 & TRL 63-92E & -4735 & 10 & -22.0 & 5738 & 73 & B \\
\hline LJ -2167 & TRL 63-92E & -4695 & 10 & -21.2 & 5625 & 57 & -14 \\
\hline LJ -2177 & TRL $63-92 \mathrm{E}$ & -4675 & 10 & -22.8 & 5782 & 58 & 143 \\
\hline $\mathrm{LJ}-2160$ & TRL 63-92E & -4655 & 10 & -22.3 & 5703 & 95 & $\mathbf{M}$ \\
\hline $\mathrm{LJ}-2170$ & TRL 63-92E & -4635 & 10 & $(-21.0)$ & 5795 & 58 & 107 \\
\hline LJ J-2165 & TRL $63-92 \mathrm{E}$ & -4615 & 10 & -21.6 & 5684 & 56 & 18 \\
\hline LJ -2173 & TRL 63-92E & -4575 & 10 & -21.9 & 5802 & 59 & 31 \\
\hline LJ -1543 & TRL، 63-92 & -4373 & 34 & -22.8 & 5433 & 84 & B \\
\hline LJ -1503 & TRL $63-92$ & -4325 & 10 & -22.3 & 5334 & 59 & 9 \\
\hline LJ-1506 & TRL 63-92 & -4305 & 10 & -21.8 & 5387 & 60 & -13 \\
\hline LJ-1505 & TRL 63-92 & -4265 & 10 & -21.8 & 5404 & 104 & M \\
\hline LJ-3548 & TRL 71-52 & -4235 & 10 & -19.4 & 5390 & 48 & -9 \\
\hline LJ-1504 & TRL 63-92 & -4225 & 10 & -21.8 & 5241 & 103 & $\mathbf{M}$ \\
\hline LJ -3547 & TRL 71-52 & -4215 & 10 & -20.3 & 5334 & 47 & -46 \\
\hline LJ-1553 & TRL $63-92$ & -4205 & 10 & -22.8 & 5174 & 64 & 102 \\
\hline LJ-3546 & TRL 71-52 & -4205 & 10 & -21.0 & 5305 & 47 & -93 \\
\hline LJ-3554 & TRL 71-52 & -4185 & 10 & -21.4 & 5327 & 52 & 80 \\
\hline LJ -1542 & TRL 63-92 & -4179 & 22 & -23.1 & 5269 & 61 & 67 \\
\hline LJ J-3553 & TRL 71-52 & -4175 & 10 & -20.4 & 5327 & 47 & -22 \\
\hline LJ-3552 & TRL 71-52 & -4155 & 10 & -20.6 & 5286 & 53 & 26 \\
\hline LJ -3555 & TRL $71-52$ & -4135 & 10 & -21.0 & 5308 & 47 & 71 \\
\hline LJ-2632 & TRL 71-52 & -4125 & 10 & -20.6 & 5150 & 54 & -147 \\
\hline LJ -3556 & TRL 71-52 & -4115 & 10 & -20.6 & 5370 & 47 & -39 \\
\hline LJ-2633 & TRL 71-52 & -4105 & 10 & -20.9 & 5167 & 53 & -37 \\
\hline LJ-1302 & TRL 65-F131 & -4077 & 52 & -28.7 & 5209 & 59 & -100 \\
\hline
\end{tabular}


Appendix 1 (continued)

\begin{tabular}{|c|c|c|c|c|c|c|c|}
\hline 1. & 2. & 3. & 4. & 5. & 6. & 7 & 8. \\
\hline LJ-2757 & TRL 71-52 & -4065 & 10 & -21.3 & 5272 & 51 & -177 \\
\hline LJ-1307 & TRI $65-\mathrm{F} 131$ & -4035 & 35 & -27.3 & 5186 & 81 & M \\
\hline LJ -2818 & TRL $71-52$ & -4025 & 10 & -21.1 & 5203 & 50 & 73 \\
\hline LJ J-2820 & TRL $71-52$ & -4005 & 10 & -21.5 & 5300 & 51 & 49 \\
\hline LJ-1305 & TRL 65-F131 & -4005 & 26 & -25.6 & 5204 & 59 & 8 \\
\hline LJ -2817 & TRL $71-52$ & -3985 & 10 & -20.4 & 5264 & 50 & -147 \\
\hline LJ-2821 & TRL 71-52 & -3965 & 10 & -21.0 & 5171 & 50 & 54 \\
\hline LJ-2819 & TRL 7 I-52 & -3945 & 10 & -20.4 & 5210 & 50 & -60 \\
\hline LJ-1306 & TRL 65-F131 & -3938 & 26 & -24.6 & 5203 & 82 & $\mathbf{M}$ \\
\hline LJ J -2825 & TRL $71-52$ & -3905 & 10 & -21.3 & 5101 & 49 & 25 \\
\hline LJ-2826 & TRL $71-52$ & -3885 & 10 & -20.9 & 4979 & 49 & 57 \\
\hline LJ J-2828 & TRL $71-52$ & -3865 & 10 & -21.2 & 5024 & 49 & -71 \\
\hline LJ J-2830 & TRL $71-52$ & -3845 & 10 & -20.8 & 5117 & 49 & -140 \\
\hline LJ-1304 & TRL 65-F131 & -3822 & 17 & -23.7 & 5057 & 59 & -19 \\
\hline LJ-2832 & TRL $71-52$ & -3805 & 10 & -20.3 & 5031 & 49 & 31 \\
\hline LJ-1299 & TRL $63-34$ & -3790 & 20 & -26.3 & 4945 & 58 & -23 \\
\hline LJ -3504 & TRL 71-52 & -3785 & 10 & -20.7 & 4865 & 46 & -40 \\
\hline LJ -1301 & TRL $63-34$ & -3772 & 24 & -30.1 & 5027 & 56 & -113 \\
\hline LJ -3505 & TRL $71-52$ & -3765 & 10 & -19.4 & 5001 & 46 & -239 \\
\hline LJ -3526 & TRL $71-52$ & -3745 & 10 & -20.8 & 4842 & 46 & 49 \\
\hline LJ -3529 & TRL $71-52$ & -3725 & 10 & -20.0 & 4951 & 46 & 3 \\
\hline LJ-3512 & TRL 71-52 & -3705 & 10 & -20.2 & 4983 & 45 & -129 \\
\hline LJ $J 1300$ & TRL 63-34 & -3700 & 20 & -25.5 & 4927 & 58 & 8 \\
\hline LJ -3520 & TRL $71-52$ & -3685 & 10 & -20.3 & 4874 & 46 & -17 \\
\hline LJ-3521 & TRL $71-52$ & -3665 & 10 & -20.0 & 4893 & 45 & 67 \\
\hline LJ -3513 & TRL $71-52$ & -3645 & 10 & -20.1 & 4893 & 46 & I \\
\hline LJ J-1298 & TRL 63-34 & -3635 & 10 & -22.9 & 4840 & 56 & 124 \\
\hline LJ J-1340 & TRL 63-34 & -3625 & 10 & -35.1 & 4717 & 55 & -75 \\
\hline LJ -3510 & TRL 71-52 & -3625 & 10 & -20.3 & 4767 & 45 & 41 \\
\hline LJ-1341 & TRL $63-34$ & -3605 & 10 & -26.3 & 4759 & 57 & 62 \\
\hline LJ -3511 & TRL $71-52$ & -3605 & 10 & -20.3 & 4758 & 45 & -97 \\
\hline LJ-3522 & TRL $71-52$ & -3585 & 10 & -20.6 & 4779 & 45 & -142 \\
\hline LJ-3523 & TRL $71-52$ & -3565 & 10 & -20.6 & 4748 & 45 & -10 \\
\hline LJ -3525 & IRI $71-52$ & -3545 & 10 & -20.6 & 4918 & 46 & 26 \\
\hline LJ-3528 & TRL $71-52$ & -3525 & 10 & $-2 \mathrm{i} .1$ & 4732 & 45 & -53 \\
\hline LJ-3524 & TRL $71-52$ & -3505 & 10 & -20.6 & 4752 & 45 & -92 \\
\hline LJ-1286 & TRL $63-89$ & -3495 & 10 & -23.9 & 4710 & 58 & 33 \\
\hline LJJ-3527 & TRL 71-52 & -3485 & 10 & -20.8 & 4673 & 46 & -93 \\
\hline LJ J 1705 & 'TRL 63-89 & -3485 & 10 & -22.1 & 4632 & 53 & 23 \\
\hline LJ-1288 & TRL $63-89$ & -3465 & 10 & -26.8 & 4628 & 57 & 22 \\
\hline LJ J-1294 & TRL 63-89 & -3455 & 10 & -24.9 & 4626 & 55 & 1 \\
\hline LJ J 1342 & TRL 63-89 & -3445 & 10 & -22.9 & 4660 & 56 & 45 \\
\hline LJ -1290 & TRL 63-89 & -3435 & 10 & -2.3 .4 & 4636 & 55 & -12 \\
\hline LJ-1322 & TRL $63-89$ & -3415 & 10 & -24.0 & 4715 & 55 & 3 \\
\hline LJ -1289 & TRL $63-89$ & -3405 & 10 & -23.8 & 4637 & 55 & -102 \\
\hline LJ-3929 & TRL $71-52$ & -3355 & 10 & -21.2 & 4560 & 46 & 52 \\
\hline LJ-3934 & TRL $71-52$ & -3335 & 10 & -21.0 & 4469 & 46 & -92 \\
\hline LJ J -1585 & TRL $63-34$ & -3325 & 10 & -21.9 & 4448 & 65 & 99 \\
\hline LJJ-3937 & IRL 71-52 & -3315 & 10 & -20.6 & 4434 & 45 & -13 \\
\hline LJ J3932 & TRL $71-52$ & -3301 & 10 & -21.2 & 4485 & 46 & 115 \\
\hline LJ-1287 & TRL 63-89 & -3290 & 20 & -30.4 & 4440 & 56 & -53 \\
\hline LJ -1529 & TRL 63-34 & -3275 & 10 & -22.6 & 4392 & 59 & -6 \\
\hline LJ-1526 & TRL 63-34 & -3255 & 10 & -21.8 & 4332 & 60 & -9 \\
\hline LJ -1276 & TRL $63-89$ & -3220 & 20 & -23.8 & 4444 & 57 & -12 \\
\hline LJ-1524 & TRL $63-34$ & -3175 & 10 & -22.2 & 4434 & 56 & 60 \\
\hline LJ -1489 & TRL 63.34 & -3125 & 10 & -23.3 & 4365 & 56 & 0 \\
\hline LJ'-1487 & TRL 63-34 & -3115 & 30 & -21.6 & 4399 & 58 & 34 \\
\hline$L J-1275$ & TRL 63-89 & -3105 & 10 & -30.7 & 4371 & 77 & M \\
\hline LJ J-1497 & TRL 63-89 & -3065 & 10 & -23.2 & 4375 & 56 & 84 \\
\hline LJ-1512 & TRL 63-89 & -3045 & 10 & -22.5 & 4436 & 56 & -59 \\
\hline
\end{tabular}


APpendix 1 (continued)

\begin{tabular}{|c|c|c|c|c|c|c|c|}
\hline 1. & 2. & 3. & 4. & 5. & 6. & 7. & 8. \\
\hline LJ-1494 & TRL 63-89 & -3025 & 10 & -23.7 & 4419 & 56 & 153 \\
\hline LJ-1704 & TRL $63-34$ & -3005 & 10 & -20.9 & 4363 & 47 & 20 \\
\hline Li-12.68 & TRL $63-89$ & -2995 & 10 & -22.7 & 4337 & 57 & -32 \\
\hline LJ-1496 & TRL $63-89$ & -2985 & 10 & -22.7 & 4315 & 55 & -21 \\
\hline LJ-1662 & TRL $63-89$ & -2965 & 10 & -22.2 & 4330 & 50 & 3 \\
\hline LJ-1498 & TRL $63-89$ & -2945 & 10 & -22.7 & 4323 & 55 & 115 \\
\hline LJ-1663 & TRL $63-89$ & -2925 & 10 & -22.0 & 4220 & 50 & 95 \\
\hline LJ-1495 & TRL $63-89$ & -2905 & 10 & -23.1 & 4217 & 55 & -75 \\
\hline L. $1-1267$ & TRL $63-89$ & -2875 & 10 & -23.2 & 4184 & 78 & M \\
\hline LJ-1511 & TRL $63-89$ & -2825 & 10 & -22.0 & 3977 & 54 & -18 \\
\hline LJ -1510 & TRL $63-89$ & -2805 & 10 & -21.6 & 4125 & 54 & -30 \\
\hline LJ-1692 & TRL $63-89$ & -2785 & 10 & -23.2 & 4113 & 51 & 43 \\
\hline 1.1270 & TRL $63-89$ & -2780 & 20 & -26.0 & 4093 & 56 & 19 \\
\hline LJ-1584 & TRL 63-89 & -2745 & 10 & -22.5 & 4088 & 63 & -30 \\
\hline LJ-1519 & TRL $63-89$ & -2718 & 25 & -22.1 & 4057 & 73 & B \\
\hline LJ-1269 & TRL $63-89$ & -2680 & 20 & -22.3 & 4087 & 59 & 12 \\
\hline LJ-1313 & TRL $63-89$ & -2645 & 10 & -23.4 & 4021 & 53 & -53 \\
\hline LJ-1319 & TRL $63-89$ & -2613 & 35 & -32.6 & 4137 & 76 & M \\
\hline LJ-1316 & TRL $63-89$ & -2585 & 10 & -23.6 & 4103 & 54 & -109 \\
\hline LJ -1320 & TRL $63-89$ & -2565 & 10 & -27.5 & 4050 & 53 & -58 \\
\hline LJ-1321 & TRL $63-89$ & -2545 & 10 & -25.8 & 3978 & 53 & 67 \\
\hline LJ-1327 & TRL $63-89$ & -2485 & 10 & -22.7 & 4019 & 76 & M \\
\hline LJ-1326 & TRL $63-89$ & -2465 & 10 & -27.6 & 3949 & 75 & M \\
\hline LJ-1333 & TRL $63-89$ & -2445 & 10 & -36.2 & 3851 & 52 & -76 \\
\hline LJ-1325 & TRL $63-89$ & -2425 & 10 & -23.1 & 4007 & 76 & M \\
\hline LJ-1328 & TRL $63-89$ & -2405 & 10 & -24.0 & 3926 & 55 & -33 \\
\hline LJ-1185 & TRL $63-53$ & -2362 & 7 & -22.0 & 3856 & 96 & B \\
\hline LJ-1187 & TRL $63-53$ & -2345 & 7 & -21.8 & 3734 & 58 & 97 \\
\hline LJ-3852 & TRL $63-53$ & -2324 & 4 & $(-24.9)$ & 3666 & 84 & M \\
\hline LJ-3831 & TRL $63-53$ & -2315 & 6 & -19.4 & 3745 & 46 & 33 \\
\hline LJ-1198 & TRL $63-53$ & -2308 & 5 & -23.3 & 3770 & 55 & -25 \\
\hline LJ-3836 & TRL $63-53$ & -2308 & 4 & -20.0 & 3819 & 45 & 62 \\
\hline LJ-1197 & TRL $63-43$ & -2302 & 28 & -25.4 & 3837 & 75 & M \\
\hline LJ-3834 & TRL 63-53 & -2300 & 4 & -19.9 & 3751 & 54 & 159 \\
\hline LJ-1193 & TRL 63-53 & -2298 & 5 & -22.2 & 3727 & 54 & 44 \\
\hline LJ-3835 & TRL $63-53$ & -2284 & 4 & -20.0 & 3756 & 45 & 62 \\
\hline LJ-1194 & TRL $63-43$ & -2282 & 23 & -23.7 & 3769 & 54 & 18 \\
\hline LJ-1186 & TRL $63-53$ & -2282 & 5 & -22.0 & 3749 & 59 & -161 \\
\hline LJ-2951 & TRL $63-53$ & -2279 & 3 & -20.6 & 3710 & 41 & -119 \\
\hline LJ-3240 & TRL 63-53 & -2275 & 4 & -20.1 & 3755 & 42 & 3 \\
\hline LJ-2415 & TRL 63-53 & -2272 & 16 & -19.7 & 3683 & 47 & -8 \\
\hline LJ-3238 & TRL $63-53$ & -2268 & 5 & -20.5 & 3754 & 33 & 17 \\
\hline LJ-3832 & TRL, $63-53$ & -2268 & 4 & -19.7 & 3759 & 46 & -90 \\
\hline LJ-3236 & TRL 63-53 & -2252 & 5 & -20.0 & 3679 & 42 & 56 \\
\hline LJ-3242 & TRL $63-53$ & -2248 & 5 & -20.3 & 3779 & 42 & -114 \\
\hline LJ-1204 & TRL $63-53$ & -2246 & 8 & -22.4 & 3823 & 75 & M \\
\hline LJ-3023 & TRL $63-53$ & -2245 & 10 & -20.3 & 3774 & 42 & 31 \\
\hline LJ -3830 & TRL $63-53$ & -2244 & 4 & -20.0 & 3666 & 47 & -10 \\
\hline LJ-3235 & TRL $63-53$ & -2238 & 5 & -19.4 & 3803 & 42 & 203 \\
\hline LJ-3827 & TRL 63-53 & -2236 & 4 & -20.2 & 3795 & 45 & 28 \\
\hline LJ-3237 & TRL 63-53 & -2222 & 5 & -20.4 & 3708 & 42 & -70 \\
\hline LJ-3851 & TRL $63-53$ & -2220 & 4 & -19.8 & 3703 & 59 & B \\
\hline LJ-2950 & TRL $63-53$ & -2214 & 3 & -21.5 & 3719 & 41 & 75 \\
\hline LJ-3829 & TRL $63-53$ & -2213 & 6 & -19.9 & 3751 & 46 & 57 \\
\hline LJ-2924 & TRL $63-53$ & -2210 & 4 & -21.2 & 3726 & 42 & -129 \\
\hline LJ-2952 & TRL $63-53$ & -2207 & 3 & -21.2 & 3665 & 41 & 119 \\
\hline LJ-2923 & TRL $63-53$ & -2204 & 3 & $-20 . \bar{T}$ & 3729 & 41 & -25 \\
\hline LJ-3239 & TRL $63-53$ & -2202 & 5 & -20.4 & 3736 & 41 & 119 \\
\hline LJ-2947 & TRL 63-53 & -2201 & 3 & -21.8 & 3691 & 40 & -46 \\
\hline LJ-3833 & TRL $63-53$ & -2197 & 4 & -20.2 & 3713 & 45 & -88 \\
\hline
\end{tabular}


Appendix 1 (continued)

\begin{tabular}{|c|c|c|c|c|c|c|c|}
\hline 1. & 2. & 3. & 4. & 5. & 6. & 7. & 8. \\
\hline LJ-3241 & TRL 63-53 & -2190 & 5 & -20.5 & 3650 & 42 & 14 \\
\hline LJ -3828 & TRL 63-53 & -2188 & 4 & -19.1 & 3660 & 42 & 0 \\
\hline LJ-1180 & TRL 63-53 & -2186 & 20 & -23.2 & 3892 & 83 & B \\
\hline LJ J-3022 & TRL 63-53 & -2178 & 10 & -20.2 & 3663 & 43 & -61 \\
\hline LJ-3021 & TRL 63-53 & -2117 & 5 & -20.5 & 3717 & 43 & -30 \\
\hline LJ'-1199 & TRL 63-53 & -2105 & 10 & -24.0 & 3629 & 54 & -34 \\
\hline LJ-1181 & TRL 63-53 & -2074 & 8 & -21.9 & 3670 & 59 & 24 \\
\hline LJ-1188 & TRL 63-53 & -2044 & 18 & -23.9 & 3566 & 75 & $\mathbf{M}$ \\
\hline LJ-1183 & TRL $63-53$ & -2025 & 10 & -23.1 & 3583 & 59 & -122 \\
\hline L.1-1483 & IRL, 63-89 & -2005 & 10 & -22.0 & 3635 & 55 & -34 \\
\hline LJ-1694 & TRL $63-89$ & -1945 & 10 & -20.9 & 3574 & 49 & 6 \\
\hline LJ-1475 & TRL $63-89$ & -1925 & 10 & -22.3 & 3482 & 53 & -15 \\
\hline LJ -1589 & TRL $63-89$ & -1905 & 10 & $(-22.0)$ & 3643 & 78 & M \\
\hline LJ J-1476 & TRL $63-89$ & -1885 & 10 & -22.6 & 3515 & 53 & -3 \\
\hline LJ-1592 & TRL 63-89 & -1865 & 10 & -21.5 & 3501 & 77 & M \\
\hline LJ-1472 & TRL 63-89 & -1845 & 10 & -22.1 & 3558 & 67 & B \\
\hline LJ J 1596 & TRL $63-89$ & -1825 & 10 & -21.4 & 3490 & 77 & $\vec{M}$ \\
\hline LJ-1473 & TRI，63-89 & -1805 & 10 & -22.3 & 3469 & 53 & -6 \\
\hline LJ-1467 & TRL $63-89$ & -1785 & 10 & -22.4 & 3524 & 57 & 3 \\
\hline LJ-1853 & TRL $63-89$ & -1775 & 10 & -21.3 & 3483 & 63 & -30 \\
\hline LJ -1850 & TRL $63-89$ & -1755 & 10 & -21.5 & 3475 & 63 & 17 \\
\hline LJ-1468 & TRI, 63-89 & -1745 & 10 & -22.1 & 3366 & 74 & $\mathrm{~B}$ \\
\hline LJ-1852 & TRL 63-89 & -1735 & 10 & -21.4 & 3387 & 62 & -165 \\
\hline LJ'-1474 & TRI, 63-89 & -1725 & 10 & -22.1 & 3427 & 53 & 10 \\
\hline LJ-1851 & TRL $63-89$ & -1715 & 10 & -21.4 & 3421 & 62 & -10 \\
\hline LJ J I 469 & TRL $63-89$ & -1705 & 10 & -21.7 & 3420 & 75 & $B$ \\
\hline LJ J 1857 & TRL 63-89 & -1695 & 10 & -21.0 & 3429 & 62 & 27 \\
\hline LJ-1583 & TRL $63-89$ & -1665 & 10 & -21.1 & 3246 & 59 & -21 \\
\hline L.J-1839 & TRL $63-89$ & -1655 & 10 & -21.8 & 3320 & 62 & 101 \\
\hline LJ -1465 & TRL $63-89$ & -1645 & 10 & -21.5 & 3321 & 57 & 26 \\
\hline LJ-1537 & TRI, 63-89 & -1625 & 10 & -21.8 & 3325 & 55 & -29 \\
\hline LJ -1845 & TRL $63-89$ & -1615 & 10 & -22.6 & 3246 & 61 & 58 \\
\hline $\mathrm{LJ}-1847$ & TRI $63-89$ & -1595 & 10 & -21.2 & 3266 & 62 & -88 \\
\hline LJ-1693 & TRL 63-89 & -1585 & 10 & -21.0 & 3179 & 48 & -71 \\
\hline LJ'-1258 & TRL $63-43$ & -1580 & 20 & -27.3 & 3236 & 78 & $\mathrm{~B}$ \\
\hline LJ J 1840 & TRI, 63-89 & -1575 & 10 & -21.7 & 3252 & 61 & 12 \\
\hline LJ'-1531 & TRL 63-89 & -1555 & 30 & -22.4 & 3152 & 69 & B \\
\hline LJ-1846 & TRL 63-89 & -1555 & 10 & -21.6 & 3189 & 61 & 33 \\
\hline $\mathbf{L j}-1466$ & TRL $63-89$ & -1545 & 10 & -21.1 & 3275 & 57 & 35 \\
\hline LJ -1838 & TRL 63-89 & -1515 & 10 & -21.7 & 3160 & 61 & 88 \\
\hline LJ -1828 & TRL 63-89 & -1505 & 10 & -22.0 & 3212 & 61 & -23 \\
\hline LJ -1525 & TRL 63-89 & -1505 & 10 & -21.5 & 3149 & 55 & 76 \\
\hline LJ-1253 & TRL $63-430$ & -1500 & 20 & $(-23.0)$ & 3147 & 78 & B \\
\hline LJ-1833 & TRL $63-89$ & -1495 & 10 & -21.5 & 3100 & 61 & 5 \\
\hline $1.1-1482$ & TRI, 63-89 & -1485 & 10 & -21.9 & 3142 & 52 & -4 \\
\hline LJ -1530 & TRL $63-89$ & -1479 & 17 & -22.6 & 3190 & 52 & -42 \\
\hline LJ'-1837 & TRL $63-89$ & -1475 & 10 & -21.2 & 3102 & 61 & 122 \\
\hline LJ-1834 & TRL $63-89$ & -1455 & 10 & -21.5 & 3211 & 61 & -63 \\
\hline $1.1-1481$ & TRL $63-89$ & -1445 & 10 & -22.0 & 3154 & 52 & 150 \\
\hline LJ -1259 & TRI $63-43$ & -1412 & 15 & -22.8 & 3167 & 53 & -27 \\
\hline LJ'-1703 & TRL 63-89 & -1405 & 10 & -20.8 & 3011 & 62 & $\mathrm{~B}$ \\
\hline LJ'-1903 & TRL 63-43 & -1395 & 10 & -21.8 & 3061 & 79 & M \\
\hline LJ-1885 & TRL $\quad 63-43$ & -1385 & 10 & -22.6 & 3003 & 91 & B \\
\hline LJ-1882 & TRL $63-43$ & -1375 & 10 & -22.1 & 3044 & 59 & -61 \\
\hline LJ-1894 & TRL $63-43$ & -1365 & 10 & -22.6 & 2927 & 59 & 80 \\
\hline LJ-1884 & TRL 63-43 & -1355 & 10 & -22.3 & 3012 & 59 & -61 \\
\hline LJ-2399 & TRL $63-43$ & -1345 & 10 & -22.0 & 2937 & 46 & -125 \\
\hline LJ-1893 & TRL 63-43 & -1325 & 10 & -22.2 & 3095 & 60 & -77 \\
\hline $\mathrm{LJ}-1881$ & TRL $63-43$ & -1315 & 10 & -22.7 & 2943 & 59 & 95 \\
\hline $\mathrm{LJ}-1870$ & TRL $63-43$ & -1285 & 10 & -21.7 & 2976 & 91 & B \\
\hline
\end{tabular}


Appendix 1 (continued)

\begin{tabular}{|c|c|c|c|c|c|c|c|}
\hline 1. & 2. & 3. & 4 & 5. & 6. & 7 & 8. \\
\hline LJ -1345 & TRL 63-43 & -1250 & 40 & -26.5 & 2980 & 51 & 80 \\
\hline LJ'-1872 & TRL $63-43$ & -1235 & 10 & -22.9 & 2970 & 60 & 27 \\
\hline LJ-1871 & TRL 63-43 & -1225 & 10 & -22.0 & 2973 & 60 & 17 \\
\hline LJ -1254 & TRL $63-43$ & -1215 & 30 & $(-23.0)$ & 2874 & 77 & B \\
\hline LJ-1865 & TRL $63-43$ & -1205 & 10 & -21.1 & 2862 & 60 & 11 \\
\hline LJ-1876 & TRL $63-43$ & -1195 & 10 & -22.4 & 2933 & 60 & -9 \\
\hline $\mathbf{L J}-1548$ & TRL 63-43 & -1185 & 10 & -22.7 & 2860 & 52 & 6 \\
\hline LJ J-1861 & TRL 63-43 & -1175 & 10 & $(-21.0)$ & 2924 & 60 & -80 \\
\hline LJ J-1878 & TRL 63-43 & -1165 & 10 & -21.3 & 2989 & 59 & -41 \\
\hline LJ-1858 & TRL $63-43$ & -1155 & 10 & -22.1 & 2985 & 60 & 41 \\
\hline $\mathrm{LJ}-1873$ & TRL 63-43 & -1145 & 10 & -21.8 & 3032 & 61 & -88 \\
\hline LJ J-1877 & TRL 63-43 & -1135 & 10 & -22.2 & 2945 & 59 & 74 \\
\hline LJ-1249 & TRL $63-43 \mathrm{C}$ & -1125 & 30 & -26.1 & 2943 & 52 & -70 \\
\hline LJ -1859 & TRL $63-43$ & -1125 & 10 & -21.8 & 2983 & 60 & 41 \\
\hline LJ -1860 & TRL $63-43$ & -1115 & 10 & -21.6 & 2957 & 61 & 102 \\
\hline LJ'-1205 & TRL $63-43$ & -1085 & 30 & -23.5 & 2831 & 56 & -45 \\
\hline LJ -1550 & TRL 63-43 & -1065 & 10 & -21.6 & 2787 & 51 & -55 \\
\hline LJ -1556 & TRL 63-43 & -1025 & 10 & -22.2 & 2790 & 53 & 30 \\
\hline LJ-1549 & TRI $63-43$ & -1005 & 10 & -22.0 & 2819 & 51 & -6 \\
\hline LJ-1209 & TRL $63-43$ & -988 & 25 & -24.4 & 2768 & 51 & -42 \\
\hline LJ-1554 & TRL 63-43 & -985 & 10 & -22.3 & 2752 & 54 & -66 \\
\hline LJ-1695 & TRL 63-43 & -975 & 10 & -22.2 & 2681 & 46 & -123 \\
\hline $\mathrm{LJ}-1250$ & TRL $63-43$ & -962 & 25 & -27.0 & 2726 & 51 & 76 \\
\hline LJ -1208 & TRL $63-43$ & -938 & 25 & -22.9 & 2850 & 52 & 22 \\
\hline LJ -1564 & TRL 63-43 & -905 & 10 & -21.4 & 2798 & 54 & 27 \\
\hline LJ-1557 & TRL $63-43$ & -895 & 10 & -22.7 & 2708 & 56 & -127 \\
\hline LJ -1210 & TRL 63-43 & -888 & 25 & -23.2 & 2677 & 52 & 144 \\
\hline LJ-1566 & TRL 63-43 & -885 & 10 & -21.4 & 2684 & 53 & 7 \\
\hline LJ-1559 & TRL $63-43$ & -879 & 18 & -22.8 & 2774 & 75 & $\mathrm{~B}$ \\
\hline LJ-1696 & TRL 63-43 & -865 & 10 & -21.5 & 2639 & 47 & 12 \\
\hline LJ-1248 & TRL 63-43 & -862 & 25 & -23.0 & 2663 & 51 & -21 \\
\hline LJ-1558 & TRL 63-43 & -855 & 10 & -23.5 & 2775 & 57 & 7 \\
\hline LJ-1590 & TRL 63-43 & -845 & 10 & -21.8 & 2740 & 74 & $\mathbf{M}$ \\
\hline LJ-1207 & TRL 63-43 & -834 & 31 & -25.4 & 2702 & 75 & B \\
\hline LJ J 1591 & TRL 63-43 & -825 & 10 & -22.2 & 2718 & 73 & $\mathrm{M}$ \\
\hline LJ -1565 & TRL 63-43 & -815 & 10 & -22.1 & 2597 & 53 & 41 \\
\hline $\mathrm{LJ}-1567$ & TRL 63-43 & -805 & 10 & -21.7 & 2579 & 53 & -23 \\
\hline LJ -1570 & TRL $63-43$ & -785 & 10 & -22.2 & 2584 & 56 & -18 \\
\hline LJ J-3913 & TRL $63-43$ & -785 & 10 & $(-21.8)$ & 2454 & 64 & $\mathbf{M}$ \\
\hline LJ J-3915 & TRL 63-43 & -775 & 10 & -21.8 & 2486 & 41 & 27 \\
\hline LJ J -3911 & TRL 63-43 & -765 & 10 & -21.7 & 2465 & 41 & 38 \\
\hline LJ J-3912 & TRL $63-43$ & -755 & 10 & -21.6 & 2398 & 41 & -83 \\
\hline LJ J-3907 & TRI. 63-43 & -745 & 10 & -21.8 & 2414 & 41 & 35 \\
\hline LJ-3914 & TRL 63-43 & -735 & 10 & -21.4 & 2496 & 41 & -4 \\
\hline LJ -1572 & TRL 63-43 & -725 & 10 & -22.2 & 2423 & 55 & 25 \\
\hline LJ J-1213 & TRL $62-68$ & -712 & 25 & -23.2 & 2416 & 50 & -40 \\
\hline LJ J-1595 & TRL $63-43$ & -705 & 10 & -22.1 & 2444 & 73 & $\mathbf{M}$ \\
\hline LJ -3910 & TRL $63-43$ & -705 & 10 & -22.4 & 2382 & 41 & -14 \\
\hline LJ -3908 & TRL $63-43$ & -695 & 10 & -22.0 & 3435 & 41 & -9 \\
\hline LJ-1283 & TRL 62-68 & -685 & 20 & -22.7 & 2518 & 51 & -98 \\
\hline LJ-1573 & TRL $63-43$ & -685 & 10 & -22.1 & 2439 & 55 & 124 \\
\hline LJ -3906 & TRL $63-43$ & -685 & 10 & -22.0 & 2487 & 42 & 23 \\
\hline LJ-1697 & TRL $63-43$ & -665 & 10 & -21.7 & 2418 & 46 & -31 \\
\hline LJ J 1571 & TRL $63-43$ & -645 & 10 & $(-22.0)$ & 2508 & 55 & 9 \\
\hline LJ-3909 & TRL 63-43 & -645 & 10 & -22.4 & 2395 & 42 & 86 \\
\hline LJ-1574 & TRL 63-43 & -625 & 10 & -22.0 & 2517 & 55 & 51 \\
\hline LJ-1284 & T RL $62-68$ & -610 & 20 & -22.4 & 2514 & 69 & $\mathbf{M}$ \\
\hline LJ-1577 & TRL 63-43 & -605 & 10 & -22.2 & 2358 & 83 & $\mathbf{M}$ \\
\hline LJ J-3903 & TRL 63-43 & -595 & 10 & -21.7 & 2441 & 42 & -82 \\
\hline LJ J-1575 & TRL 63-43 & -585 & 10 & -22.1 & 2294 & 55 & -87 \\
\hline
\end{tabular}


Appendix 1 (continued)

\begin{tabular}{|c|c|c|c|c|c|c|c|}
\hline 1. & 2. & 3. & 4. & 5. & 6. & 7. & 8. \\
\hline LJ-3891 & TRL $63-43$ & -585 & 10 & -22.3 & 2162 & 45 & -43 \\
\hline LJ-3892 & TRL 63-43 & -575 & 10 & -21.9 & 2397 & 41 & 75 \\
\hline LJ -1578 & TRL $63-43$ & -545 & 10 & -22.4 & 2432 & 56 & 29 \\
\hline LJ -3889 & TRL $63-43$ & -545 & 10 & -22.1 & 2288 & 42 & 45 \\
\hline LJ -3900 & TRL 63-43 & -535 & 10 & -21.8 & 2374 & 42 & -4 \\
\hline LJ -1576 & TRL 63-43 & -525 & 10 & -22.0 & 2399 & 56 & -88 \\
\hline LJ-3902 & TRL 63-43 & -525 & 10 & -21.8 & 2348 & 41 & -127 \\
\hline LJ-390I & TRL 63-43 & -515 & 10 & -21.1 & 2407 & 41 & -66 \\
\hline LJ-1671 & TRL 63-43 & -495 & 10 & -22.1 & 2230 & 45 & -10 \\
\hline L.J-3890 & TRL 63-43 & -495 & 10 & -22.3 & 2333 & 46 & 102 \\
\hline LJ-3864 & Holstein & -488 & 10 & -25.2 & 2331 & 42 & -3 \\
\hline LJ -3860 & Holstein & -478 & 10 & -25.4 & 2313 & 42 & -16 \\
\hline LJ-1669 & TRL 63-43 & -475 & 10 & -21.2 & 2420 & 45 & 63 \\
\hline LJ -3865 & Holstein & -470 & 5 & -24.8 & 2274 & 46 & -74 \\
\hline LJ -3866 & Holstein & -465 & 5 & -25.1 & 2404 & 41 & 76 \\
\hline LJ -3859 & Holstein & -458 & 10 & -25.6 & 2336 & 42 & -118 \\
\hline LJ-1672 & TRL 63-43 & -455 & 10 & -21.1 & 2136 & 45 & -88 \\
\hline LJ -3863 & Holstein & -448 & 10 & -25.7 & 2334 & 42 & 19 \\
\hline LJ -3861 & Holstein & -438 & 10 & -25.7 & 2294 & 42 & -155 \\
\hline LJ -1670 & TRL 63-43 & -415 & 10 & -22.7 & 2339 & 45 & -50 \\
\hline LJ -3876 & TRL 63-43 & -405 & 10 & -22.4 & 2247 & 45 & 65 \\
\hline LJ-1678 & TRL 63-43 & -395 & 10 & -21.6 & 2243 & 45 & -70 \\
\hline LJ-3874 & TRL $63-43$ & -395 & 10 & -21.4 & 2243 & 41 & 26 \\
\hline LJ -3878 & TRL 63-43 & -385 & 10 & -21.5 & 2196 & 41 & -105 \\
\hline LJ-1682 & TRL 63-43 & -375 & 10 & -21.1 & 2145 & 45 & 9 \\
\hline LJ-3872 & TRL 63-43 & -375 & 10 & -22.0 & 2147 & 41 & -138 \\
\hline LJ-3877 & TRL 63-43 & -365 & 10 & -22.1 & 2152 & 42 & 66 \\
\hline LJ -3862 & Holstein & -358 & 10 & -25.1 & 2157 & 42 & 28 \\
\hline LJ-1679 & TRL 63-43 & -355 & 10 & -21.5 & 2171 & 45 & -2 \\
\hline LJ -3875 & TRL 63-43 & -355 & 10 & -21.3 & 2131 & 41 & -63 \\
\hline LJ-3341 & D4 Becker & -338 & 1 & -26.3 & 2141 & 39 & -40 \\
\hline LJ -3423 & D4 Becker & -336 & 1 & -25.4 & 2240 & 39 & 170 \\
\hline LJ -3342 & TRL 63-43 & -335 & 10 & -21.9 & 2141 & 38 & -51 \\
\hline LJ-1687 & TRL $63-43$ & -335 & 10 & -21.4 & 2129 & 45 & -39 \\
\hline LJ -3419 & I) 4 Becker & -334 & 1 & -26.0 & 2192 & 38 & 69 \\
\hline LJ -3415 & D4 Becker & -332 & 1 & -25.5 & 2139 & 38 & 69 \\
\hline LJ-3412 & D4 Becker & -330 & 1 & -25.4 & 2265 & 39 & -5 \\
\hline LJ J -3345 & D4 Becker & -326 & 1 & -24.1 & 2197 & 55 & $\mathbf{M}$ \\
\hline $\mathbf{L J}-3346$ & TRL 63-43 & -325 & 10 & -21.5 & 2165 & 38 & -42 \\
\hline LJ -3411 & D4 Becker & -324 & 1 & -25.3 & 2267 & 39 & -3 \\
\hline LJ -3422 & D4 Becker & -320 & 1 & -24.4 & 2257 & 39 & 208 \\
\hline LJ -3421 & TRL 63-43 & -315 & 10 & -21.9 & 2213 & 38 & 144 \\
\hline LJ-1680 & TRL 63-43 & -315 & 10 & -21.2 & 2146 & 45 & 13 \\
\hline LJ-1219 & TRL $62-68$ & -312 & 25 & -21.8 & 2305 & 71 & B \\
\hline LJ -3413 & TRL 63-43 & -305 & 10 & -21.4 & 2178 & 39 & -13 \\
\hline LJ -3414 & D4 Becker & -300 & 1 & -23.5 & 2151 & 38 & -39 \\
\hline LJ -3340 & TRL 63-43 & -295 & 10 & -21.7 & 2218 & 39 & 154 \\
\hline LJ-1686 & TRL 63-43 & -295 & 10 & -20.7 & 2176 & 45 & 44 \\
\hline LJ -3339 & D4 Becker & -295 & 1 & -24.5 & 2215 & 38 & -112 \\
\hline LJ J-3333 & D4 Becker & -289 & 1 & -24.0 & 2221 & 38 & 74 \\
\hline LJ-3332 & TRL 63-43 & -285 & 10 & -21.5 & 2215 & 38 & -55 \\
\hline $\mathrm{LJ}-3416$ & TRL 63-43 & -275 & 10 & -21.3 & 2175 & 38 & -57 \\
\hline $\mathrm{LJ}-1681$ & TRL 63-43 & -275 & 10 & -21.5 & 2195 & 45 & -59 \\
\hline LJ J-3343 & D4 Becker & -268 & 1 & -23.9 & 2168 & 38 & -103 \\
\hline LJ-3344 & TRL 63-43 & -265 & 10 & -21.6 & 2174 & 39 & -135 \\
\hline LJ-3331 & D4 Becker & -258 & 1 & -23.4 & 2178 & 39 & 26 \\
\hline LJ -3418 & TRL 63-43 & -255 & 10 & -22.4 & 2246 & 39 & 66 \\
\hline LJ -3334 & TRL 63-43 & -245 & 10 & -22.5 & 2191 & 42 & 16 \\
\hline LJ -3335 & D4 Becker & -240 & 1 & -24.6 & 2221 & 38 & 95 \\
\hline LJ -3336 & TRL 63-43 & -235 & 10 & -21.2 & 2197 & 38 & 108 \\
\hline
\end{tabular}


Appendix 1 (continued)

\begin{tabular}{|c|c|c|c|c|c|c|c|}
\hline 1. & 2. & 3. & 4. & 5. & 6. & 7. & 8. \\
\hline LJ-3338 & TRL 63-43 & -225 & 10 & -22.4 & 2198 & 38 & 62 \\
\hline LJ-1232 & TRL 62-68 & -225 & 10 & -23.2 & 2221 & 50 & 36 \\
\hline LJ-1231 & TRL 62-68 & -200 & 20 & -22.1 & 2228 & 69 & $\mathbf{M}$ \\
\hline LJ J-3420 & 1)4 Becker & -190 & 1 & -24.4 & 2196 & 38 & -36 \\
\hline LJ-1233 & TRL $62-68$ & -180 & 20 & -22.4 & 2158 & 50 & -54 \\
\hline LJ J-1230 & TRL 62-68 & -160 & 20 & -22.7 & 2119 & 50 & -39 \\
\hline LJ-1229 & TRL $62-68$ & -90 & 20 & -23.2 & 2007 & 49 & 84 \\
\hline LJ J-1216 & TRL 62-68 & -55 & 30 & -23.1 & 2057 & 49 & -19 \\
\hline LJ-1221 & TRL $62-68$ & -30 & 20 & -21.3 & 1979 & 49 & 76 \\
\hline LJ-1214 & TRL 62-68 & -10 & 20 & -22.3 & 2036 & 49 & 87 \\
\hline LJ J-2441 & TRL $71-51$ & 5 & 10 & -20.7 & 1988 & 43 & -78 \\
\hline LJ J-2444 & TRL 71-51 & 25 & 10 & -21.3 & 1861 & 44 & -224 \\
\hline $\mathbf{L J}-2443$ & TRL $71-51$ & 45 & 10 & -20.0 & 1910 & 43 & -18 \\
\hline LJ-2478 & TRL 71-5l & 85 & 10 & -21.8 & 1796 & 44 & -34 \\
\hline LJ J-2480 & TRL $71-51$ & 125 & 10 & -21.2 & 1792 & 46 & 38 \\
\hline LJ J-2624 & TRL $71-51$ & 185 & 10 & -20.1 & 1831 & 45 & -84 \\
\hline LJ J-2626 & TRL $71-51$ & 205 & 10 & -19.7 & 1857 & 44 & 100 \\
\hline LJ-2627 & TRL $71-51$ & 225 & 10 & -19.8 & 1769 & 43 & -32 \\
\hline LJ J-2622 & TRL $71-51$ & 245 & 10 & -20.3 & 1953 & 44 & 122 \\
\hline LJ -1746 & IRL $63-48$ & 285 & 10 & -20.1 & 1722 & 45 & 68 \\
\hline LJ J-237 I & TRL $63-48$ & 295 & 10 & -19.6 & 1738 & 56 & B \\
\hline LJ -1759 & TRL $63-48$ & 305 & 10 & -19.6 & 1682 & 45 & -24 \\
\hline LJ-1752 & TRL 63-48 & 315 & 10 & -19.8 & 1691 & 45 & -28 \\
\hline LJ J-2383 & TRL 63-48 & 335 & 10 & -19.6 & 1704 & 43 & -13 \\
\hline LJ -1747 & TRL $63-48$ & 345 & 10 & -20.2 & 1635 & 45 & 134 \\
\hline LJ -1756 & TRL $63-48$ & 355 & 10 & -20.7 & 1595 & 44 & 96 \\
\hline LJ-2385 & TRL 63-48 & 375 & 10 & -20.1 & 1566 & 43 & 75 \\
\hline LJ J -1751 & T'RI $63-48$ & 385 & 10 & -20.1 & 1615 & 45 & -54 \\
\hline LJ -2380 & TRL 63-48 & 395 & 10 & -19.8 & 1596 & 43 & -38 \\
\hline LJ-1758 & I'RL $63-48$ & 405 & 10 & -19.2 & $160 \mathrm{I}$ & 45 & 31 \\
\hline LJ J-2389 & TRL $63-48$ & 415 & 10 & -20.1 & 1625 & 43 & -9 \\
\hline LJ J-1748 & TRL 63-48 & 425 & 10 & -20.2 & 1572 & 45 & 77 \\
\hline LJJ-2382 & TRL 63-48 & 435 & 10 & -19.6 & 1543 & 57 & B \\
\hline LJ J 1757 & TRL 63-48 & 455 & 10 & -19.9 & 1544 & 45 & 28 \\
\hline LJ J-1750 & TRL $63-48$ & 475 & 10 & -19.8 & 1472 & 44 & -60 \\
\hline LJ J-2368 & TRL $63-48$ & 495 & 10 & -19.9 & 1358 & 43 & -83 \\
\hline LI 1749 & TRL $63-48$ & 505 & 10 & -19.9 & 1414 & 44 & -32 \\
\hline LJ-1718 & TRL $67-30$ & 705 & 10 & -21.1 & 1254 & 70 & $\mathrm{M}$ \\
\hline LJ'-1815 & TRL $67-30$ & 715 & 10 & $-2(0.8$ & 1228 & 45 & -24 \\
\hline LJ-1744 & TRL $67-30$ & 728 & 4 & -21.8 & 1266 & 44 & 19 \\
\hline LJ-1715 & TRL $67-30$ & 735 & 10 & -21.1 & 1221 & 44 & -32 \\
\hline LJ-1730 & TRL $67-30$ & 745 & 10 & -20.7 & 1204 & 44 & -134 \\
\hline LJ 1722 & IRL 67-30 & 755 & 10 & -20.7 & 1233 & 44 & 18 \\
\hline LJ-1724 & TRL $67-30$ & 775 & 10 & -20.2 & 1210 & 44 & 54 \\
\hline LJ J-1149 & TRL 63-48 & 778 & 15 & -22.4 & 1172 & 54 & 12 \\
\hline LJ-1811 & TRL $67-30$ & 785 & 10 & -21.0 & 1153 & 45 & 89 \\
\hline LJ-1714 & TRL $67-30$ & 795 & 10 & -20.3 & 1251 & 44 & -6 \\
\hline L.J-1813 & TRL $67-30$ & 805 & 10 & -21.4 & 1276 & 45 & 34 \\
\hline LJ -1721 & TRL $67-30$ & 815 & 10 & -20.6 & 1097 & 44 & -70 \\
\hline LJ-1743 & IRL $67-30$ & 821 & 3 & -22.0 & 1201 & 44 & 4 \\
\hline LJ-1723 & TRL $67-30$ & 835 & 10 & -20.2 & 1129 & 44 & 3 \\
\hline $\mathbf{L J}-1731$ & TRL $67-30$ & 845 & 10 & -21.0 & 1160 & 44 & 24 \\
\hline LJ-1720 & TRL $67-30$ & 855 & 10 & -20.7 & 1210 & 70 & $\vec{M}$ \\
\hline LJ-1814 & TRL $67-30$ & 865 & 10 & -21.2 & 1217 & 45 & 11 \\
\hline LJ J 1716 & TRL $67-30$ & 875 & 10 & -20.4 & 1075 & 44 & -32 \\
\hline LJ J-1812 & TRL $67-30$ & 885 & 10 & -20.5 & 1112 & 45 & 20 \\
\hline LJ-1719 & TRL $67-30$ & 895 & 10 & -20.7 & 1080 & 44 & 28 \\
\hline LJ J -3223 & TRL $67-3$ & 1085 & 10 & -21.2 & 830 & 37 & 102 \\
\hline LJ -3228 & TRL $67-3$ & 1095 & 10 & -21.7 & 961 & 37 & 43 \\
\hline LJ -3222 & TRL $67-3$ & 1105 & 10 & -21.5 & 909 & 36 & -43 \\
\hline
\end{tabular}


Appendix 1 (continued)

\begin{tabular}{|c|c|c|c|c|c|c|c|}
\hline 1. & 2. & 3. & 4. & 5. & 6. & 7. & 8. \\
\hline LJ -3229 & TRL 67-3 & 1115 & 10 & -21.0 & 892 & 37 & 29 \\
\hline LJ -3250 & Eschelbronn & 1120 & 2 & -24.9 & 977 & 37 & 0 \\
\hline LJ J-3224 & 'TRL 67-3 & 1125 & 10 & -21.2 & 935 & 37 & -70 \\
\hline LJ -3227 & TRL 67-3 & 1135 & 10 & -22.0 & 862 & 37 & 5 \\
\hline LJ -3249 & Eschelbronn & 1140 & 1 & -24.5 & 853 & 37 & -65 \\
\hline LJ-3226 & TRL 67-3 & 1145 & 10 & -20.9 & 864 & 36 & -79 \\
\hline LJ -3259 & Eschelbronn & 1160 & 1 & -25.6 & 801 & 36 & 118 \\
\hline LJ -3234 & TRL 67-3 & 1165 & 10 & -20.8 & 831 & 37 & 5 \\
\hline LJ J -3256 & Eschelbronn & 1170 & 1 & -25.6 & 858 & 36 & 96 \\
\hline LJ J-325l & Eschelbronn & 1180 & 1 & -26.3 & 847 & 36 & -4 \\
\hline LJ J-3225 & TRL $67-3$ & 1185 & 10 & -21.4 & 802 & 36 & -131 \\
\hline LJ -3255 & Eschelbronn & 1190 & 1 & -25.8 & 815 & 37 & 13 \\
\hline LJ -3277 & Gottlieben & 1193 & 7 & -26.5 & 838 & 37 & -100 \\
\hline LJ J-3254 & Eschelbronn & 1200 & 1 & -25.9 & 851 & 36 & 72 \\
\hline LJ-3231 & TRL 67-3 & 1205 & 10 & -21.7 & 769 & 37 & -2 \\
\hline LJ -3275 & Gottlieben & 1206 & 4 & -26.5 & 880 & 37 & 114 \\
\hline LJ J-3257 & Eschelbronn & 1210 & 1 & -26.0 & 784 & 36 & 32 \\
\hline LJ-328I & Eschelbronn & 1214 & 2 & -26.2 & 818 & 60 & B \\
\hline LJ -3276 & Gottlicben & 1216 & 4 & -26.0 & 839 & 37 & 5 \\
\hline LJ J-3260 & Eschelbronn & 1220 & I & -25.8 & 847 & 37 & -18 \\
\hline LJ-3232 & TRL 67-3 & 1225 & 10 & -21.0 & 782 & 36 & -8 \\
\hline LJ J-3253 & Gottlieben & 1230 & 1 & -25.5 & 776 & 36 & -63 \\
\hline LJ -3278 & Gottlieben & 1236 & 4 & -26.0 & 669 & 52 & $\mathbf{M}$ \\
\hline LJ -3258 & Eschelbronn & 1241 & 3 & -25.9 & 758 & 36 & 52 \\
\hline LJ -3233 & TRL $67-3$ & 1245 & 10 & -21.2 & 744 & 37 & -102 \\
\hline LJ -3261 & Gottlieben & 1252 & 4 & -26.1 & 729 & 37 & 101 \\
\hline LJ -3297 & TRL $67-3$ & 1255 & 10 & -21.0 & 696 & 36 & 37 \\
\hline LJ -3299 & TRL $67-3$ & 1265 & 10 & -21.6 & 692 & 36 & -167 \\
\hline LJ -3252 & Gottlieben & 1270 & 2 & -25.3 & 705 & 36 & 18 \\
\hline LJ-3301 & TRL $67-3$ & 1275 & 10 & -21.3 & 706 & 37 & 6 \\
\hline LJ -3230 & TRL $67-3$ & 1285 & 10 & -21.8 & 579 & 36 & -98 \\
\hline LJ -3298 & Gottlieben & 1290 & 2 & -25.6 & 673 & 36 & -9 \\
\hline LJ -3300 & Gottlicben & 1296 & 2 & -25.4 & 617 & 36 & -8 \\
\hline
\end{tabular}

Appendix 2

Floating tree-ring series

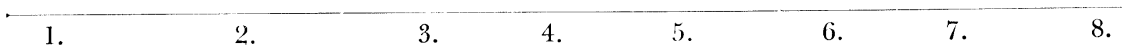

Fischbeck series, estimated zero point ca $6120 \mathrm{BC}$

$\begin{array}{llrrrrrr}\text { LJ-3490 } & \text { Fischbeck } & 30 & 5 & -26.3 & 7098 & 52 & -23 \\ \text { LJ-3489 } & \text { Fischbeck } & 90 & 5 & -25.2 & 7147 & 53 & -50 \\ \text { LJ-3488 } & \text { Fischbeck } & 270 & 5 & -24.6 & 7016 & 52 & -127 \\ \text { LJ-3491 } & \text { Fischbeck } & 330 & 5 & -24.0 & 6921 & 52 & -66\end{array}$

Donau 11 incomplete series, B Becker

$\begin{array}{llrllllr}\text { LJ-2968 } & \text { D11A } & 25 & 10 & -25.6 & 7065 & 49 & -74 \\ \text { LJ-3004 } & \text { D11A } & 55 & 10 & -25.0 & 6983 & 49 & 63 \\ \text { LJ-3000 } & \text { D11A } & 75 & 10 & -26.0 & 6904 & 50 & 26 \\ \text { LJ-3003 } & \text { D11A } & 125 & 10 & -26.6 & 6985 & 49 & 63 \\ \text { LJ-2999 } & \text { D11A } & 175 & 10 & -24.9 & 6710 & 49 & -42 \\ \text { LJ-3033 } & \text { D11A } & 195 & 10 & -27.0 & 7027 & 51 & 82 \\ \text { LJ-3034 } & \text { D11B } & 205 & 10 & -26.4 & 6215 & 48 & -71 \\ \text { LJ-2985 } & \text { D11B } & 225 & 10 & -24.8 & 6206 & 48 & -43 \\ \text { LJ-3001 } & \text { D11B } & 275 & 10 & -26.3 & 6116 & 47 & 3 \\ \text { LJ-3002 } & \text { D11B } & 325 & 10 & -25.3 & 6101 & 47 & -47 \\ \text { LJ-2984 } & \text { D11B } & 365 & 10 & -24.8 & 6070 & 47 & -79\end{array}$


APpendix 2 (continued)

\begin{tabular}{llllllll}
\hline 1. & 2. & 3. & 4. & 5. & 6. & 7. & 8. \\
\hline
\end{tabular}

Main 6 series, B Becker, estimated zero point ca 6075 BC

$\begin{array}{lllrl}\text { LJ }-3315 & \text { M6 } & 100 & 1 & -25.5 \\ \text { LJ-3305 } & \text { M6 } & 110 & 1 & -26.2 \\ \text { LJ-3307 } & \text { M6 } & 120 & 1 & -26.1 \\ \text { LJ-3316 } & \text { M6 } & 150 & 1 & -25.3 \\ \text { LJ-3480 } & \text { M6 } & 190 & 1 & -25.3 \\ \text { LJ-3309 } & \text { M6 } & 200 & 1 & -25.9 \\ \text { LJ-3476 } & \text { M6 } & 210 & 1 & -25.8 \\ \text { LJ-3320 } & \text { M6 } & 235 & 10 & -23.6 \\ \text { LJ-3317 } & \text { M6 } & 300 & 1 & -23.7 \\ \text { LJ-3478 } & \text { M6 } & 310 & 1 & -25.3 \\ \text { LJ-3319 } & \text { M6 } & 350 & 1 & -23.3 \\ \text { LJ-3318 } & \text { M6 } & 390 & 1 & -24.1 \\ \text { LJ-3477 } & \text { M6 } & 408 & 5 & -23.5 \\ \end{array}$

Donau 8 scries, B Becker, estimated zero point ca 4430 вC

\begin{tabular}{|c|c|c|c|c|c|c|c|}
\hline LJ -3057 & D8 & 5 & 1 & -26.4 & 5534 & 47 & 8 \\
\hline LJ-3010 & D8 & 12 & 1 & -26.4 & 5635 & 45 & -160 \\
\hline LJ-3039 & D8 & 17 & 1 & -26.7 & 5547 & 47 & -103 \\
\hline LJ -3077 & D8 & 19 & 1 & -25.5 & 5578 & 47 & 69 \\
\hline LJ-2968 & D8 & 23 & 1 & -24.9 & 5552 & 46 & 3 \\
\hline LJ -3147 & D8 & 26 & 1 & -25.8 & 5502 & 47 & 15 \\
\hline LJ J -3146 & D8 & 29 & 1 & -26.1 & 5509 & 48 & 72 \\
\hline LJ-3005 & D8 & 33 & 1 & -25.6 & 5508 & 47 & -46 \\
\hline LJ-3072 & D8 & 35 & 1 & -25.5 & 5448 & 47 & -146 \\
\hline LJ J-3149 & D8 & 37 & 1 & -24.3 & 5497 & 47 & 50 \\
\hline LJ -2981 & D8 & 39 & 1 & -25.1 & 5443 & 63 & $\mathbf{M}$ \\
\hline $\mathrm{LJ}-3148$ & D8 & 41 & 1 & -25.2 & 5461 & 47 & 37 \\
\hline LJ J-3037 & D8 & 44 & 1 & -25.0 & 5551 & 70 & M \\
\hline LJ -3145 & D8 & 49 & 1 & -25.0 & 5441 & 47 & -35 \\
\hline LJ J -3144 & D8 & 51 & 1 & -24.1 & 5360 & 47 & 41 \\
\hline LJ J-3060 & D8 & 53 & 1 & -24.9 & 5372 & 46 & -10 \\
\hline LJ J-3143 & D8 & 58 & 1 & -25.2 & 5365 & 47 & -53 \\
\hline LJ J -3142 & 198 & 61 & 1 & -25.3 & 5292 & 47 & -195 \\
\hline LJ J -2966 & D8 & 65 & 1 & -25.7 & 5417 & 46 & -101 \\
\hline LJ J-3062 & D8 & 72 & 1 & -24.5 & 5366 & 46 & 98 \\
\hline LJ -3500 & D8 & 75 & 5 & -24.7 & 5493 & 47 & 91 \\
\hline LJ J-3502 & D8 & 80 & 6 & -25.8 & 5426 & 47 & 20 \\
\hline LJ J-3038 & D8 & 89 & 4 & -27.5 & 5356 & 46 & 58 \\
\hline LJ-3117 & D8 & 91 & 3 & -26.7 & 5362 & 46 & -125 \\
\hline LJ -3035 & D 8 & 99 & 3 & -27.9 & 5471 & 46 & 122 \\
\hline LJ-3134 & D8 & 102 & 1 & -27.1 & 5351 & 46 & 15 \\
\hline LJ J-2967 & D8 & 104 & 1 & $-\because: 2$ & 5457 & 46 & 32 \\
\hline LJ J-3133 & D8 & 106 & 1 & -27.8 & 5363 & 45 & 177 \\
\hline LJ J-3118 & D8 & 110 & 3 & -27.4 & 5363 & 46 & 11 \\
\hline LJ J -3501 & D8 & 114 & 6 & -25.4 & 5371 & 47 & 28 \\
\hline LJ J-3014 & D8 & 115 & 1 & -26.3 & 5546 & 46 & 165 \\
\hline LJ -3168 & D8 & 118 & 1 & -26.8 & 5476 & 46 & 30 \\
\hline LJ -3075 & D8 & 120 & 1 & -26.9 & 5380 & 46 & 68 \\
\hline LJ J-3169 & D8 & 124 & 1 & -26.7 & 5374 & 46 & -5 \\
\hline LJ J-3040 & 108 & 126 & 1 & -26.9 & 5358 & 46 & -93 \\
\hline LJ-3171 & D8 & 130 & 1 & -27.1 & 5372 & 47 & 81 \\
\hline $\mathrm{LJ}-3170$ & D8 & 133 & 1 & -26.4 & 5351 & 47 & -79 \\
\hline$L J-3167$ & D8 & 136 & 1 & -26.6 & 5316 & 47 & -19 \\
\hline $\mathrm{LJ}-3012$ & D8 & 138 & 1 & -27.2 & 5328 & 45 & -167 \\
\hline LJ -3193 & D8 & 143 & 1 & --26.4 & 5346 & 47 & -17 \\
\hline LJ J-2983 & D8 & 151 & 1 & -25.9 & 5307 & 45 & 49 \\
\hline LJ -3379 & D8 & 157 & 1 & -26.4 & 5339 & 45 & 78 \\
\hline LJ-3378 & D8 & 158 & 9 & -29.6 & 5343 & 45 & -56 \\
\hline
\end{tabular}


Appendix 2 (continued)

\begin{tabular}{|c|c|c|c|c|c|c|c|}
\hline 1. & 2. & 3. & 4. & 5. & 6. & 7. & 8. \\
\hline LJ -3194 & D8 & 160 & 1 & -26.6 & 5346 & 47 & 74 \\
\hline LJ -3380 & D8 & 167 & 8 & -28.3 & 5317 & 45 & 16 \\
\hline LJ -3013 & 198 & 170 & 1 & $-2,6.5$ & 5356 & 45 & 41 \\
\hline LJ -3195 & D8 & 174 & 1 & -26.9 & 5337 & 47 & -30 \\
\hline LJ J 3376 & D8 & 174 & 7 & -28.3 & 5293 & 46 & -165 \\
\hline LJ -3377 & D8 & 179 & 3 & -27.9 & 5200 & 45 & -44 \\
\hline LJ -3076 & D8 & 179 & 1 & -27.2 & 5345 & 46 & 153 \\
\hline LJ J-3119 & 198 & 189 & 1 & -26.4 & 5261 & 46 & -16 \\
\hline LJ-3381 & D8 & 197 & 5 & -27.7 & 5376 & 46 & 35 \\
\hline LJ J -3197 & D8 & 199 & 1 & -27.8 & 5319 & 46 & -132 \\
\hline LJ-2978 & D8 & 204 & 1 & -27.1 & 5274 & 45 & -29 \\
\hline LJ-3382 & 108 & 204 & 4 & -27.1 & 5290 & 45 & -35 \\
\hline LJ -3196 & 108 & 206 & 1 & -26.9 & 5381 & 47 & 33 \\
\hline LJ-3383 & 128 & 208 & 4 & -26.6 & 5317 & 45 & -135 \\
\hline LJ -3179 & D8 & 208 & 1 & -27.1 & 5377 & 47 & 17 \\
\hline LJ -3192 & $1) 8$ & 210 & 1 & -26.8 & 5285 & 46 & 104 \\
\hline LJ -3386 & 1) 8 & 211 & 3 & -26.1 & 5337 & 45 & 15 \\
\hline LJ -3387 & D 8 & 214 & 2 & -25.4 & 5321 & 45 & -7 \\
\hline LJ -3006 & D8 & 214 & 1 & -27.7 & 5433 & 45 & 41 \\
\hline LJ J-3178 & D8 & 216 & 1 & -27.2 & 5251 & 46 & 72 \\
\hline LJ -3056 & D8 & 218 & 1 & -27.3 & 5282 & 46 & 114 \\
\hline LJ-3402 & 108 & 223 & 3 & -26.0 & 5287 & 45 & 36 \\
\hline LJ -3122 & 108 & 224 & 1 & -26.8 & 5300 & 46 & -77 \\
\hline LJ-2964 & I) 8 & 227 & 1 & -27.3 & 5247 & 44 & -8 \\
\hline LJ -2965 & D8 & 231 & 1 & -26.5 & 5230 & 44 & 95 \\
\hline LJ -3404 & D8 & 234 & 4 & -25.6 & 5298 & 45 & 78 \\
\hline LJ J 3123 & D8 & 238 & 6 & -26.4 & 5295 & 46 & -82 \\
\hline LJ -3403 & D8 & 244 & 4 & -25.9 & 5269 & 45 & -35 \\
\hline LJ J-3401 & D8 & 252 & 4 & -25.0 & 5297 & 44 & 166 \\
\hline LJ-3385 & D8 & 262 & 6 & -24.3 & 5362 & 45 & -110 \\
\hline $\mathbf{L J}-3503$ & D8 & 282 & 6 & -25.6 & 5264 & 46 & -70 \\
\hline LJ-3384 & D8 & 288 & 6 & -26.5 & 5323 & 45 & 124 \\
\hline
\end{tabular}

Neolithic master chronology (see Becker, 1978) incorporating

Thayngen-Burgaschisee series (Ferguson, Huber, and Suess, 1966), estimated zero point ca $4050 \mathrm{BC}$

\begin{tabular}{|c|c|c|c|c|c|c|c|}
\hline LJ -3610 & $199 / 12$ & 88 & 3 & -26.5 & 5159 & 46 & -265 \\
\hline LJ-1266 & Thayngen & 101 & 12 & -26.3 & 5070 & 61 & 11 \\
\hline LJ J 1262 & Thayngen & 137 & 40 & -31.1 & 5037 & 60 & 87 \\
\hline LJ -3636 & Thayngen & 139 & 15 & -24.2 & 5003 & 46 & -14 \\
\hline LJ -3598 & $109 / 12$ & 141 & 2 & -26.3 & 5058 & 46 & 130 \\
\hline LJ -3611 & $09 / 12$ & 174 & 1 & -25.3 & 5023 & 47 & 76 \\
\hline LJ -1278 & Thayngen & 182 & 10 & -26.4 & 4992 & 58 & -116 \\
\hline LJ -3609 & $109 / 12$ & 197 & 1 & -25.6 & 5029 & 46 & 42 \\
\hline LJ J 1293 & Burgasch & 207 & 40 & -35.2 & 5053 & 58 & -71 \\
\hline LJ J -1277 & Thayngen & 217 & 20 & -25.3 & 4925 & 58 & -106 \\
\hline LJ-3614 & $109 / 12$ & 222 & 1 & -25.6 & 4976 & 46 & -198 \\
\hline LJ-1261 & Thayngen & 227 & 20 & -28.0 & 5050 & 60 & -56 \\
\hline LJ -3596 & $\mathrm{D} 9 / 12$ & 257 & 2 & -24.5 & 4912 & 46 & -90 \\
\hline LJ -3625 & $109 / 12$ & 277 & 2 & -24.7 & 4963 & 46 & -78 \\
\hline $\mathrm{LJ}-1279$ & Thayngen & 287 & 20 & -27.2 & 4917 & 58 & -11 \\
\hline LJ -3626 & $1) 9 / 12$ & 299 & 2 & -24.1 & 4931 & 47 & -106 \\
\hline LJ-1265 & Thayngen & 307 & 20 & -25.6 & 4962 & 60 & 24 \\
\hline LJ-3608 & $\mathrm{D} 9 / 12$ & 335 & 2 & -23.9 & 4873 & 46 & 60 \\
\hline LJ J -3627 & $\mathrm{D} 9 / \mathrm{I} 2$ & 369 & 5 & -24.5 & 4935 & 46 & -129 \\
\hline LJ -3628 & $109 / 12$ & 396 & 3 & -23.9 & 4723 & 45 & -89 \\
\hline LJ J-3613 & $\mathrm{D9} / 12$ & 421 & 2 & -24.5 & 4742 & 45 & 9 \\
\hline LJ -3615 & $\mathrm{D9} / 12$ & 447 & 2 & -24.5 & 4727 & 45 & -52 \\
\hline LJ-3644 & D7 & 460 & 1 & -23.6 & 4881 & 46 & -173 \\
\hline$L J-3655$ & 107 & 470 & 1 & -24.3 & 4786 & 45 & -62 \\
\hline LJ J 3645 & D7 & 480 & 1 & -24.3 & 4758 & 45 & 20 \\
\hline
\end{tabular}


Appendix 2 (continued)

\begin{tabular}{|c|c|c|c|c|c|c|c|}
\hline 1. & 2. & 3. & 4. & 5. & 6. & 7. & 8. \\
\hline LJ -3667 & D7 & 490 & 1 & -24.6 & 4766 & 46 & -100 \\
\hline LJ J-3597 & D9/12 & 497 & 2 & -25.9 & 4773 & 45 & -44 \\
\hline LJ -3640 & D7 & 500 & 1 & -24.9 & 4797 & 46 & -63 \\
\hline LJ -3656 & D7 & 510 & 1 & $-2,4.5$ & 4786 & 46 & -104 \\
\hline LJ-3643 & D7 & 520 & 1 & -24.7 & 4773 & 46 & -85 \\
\hline LJ-3654 & D7 & 530 & 1 & -24.6 & 4733 & 45 & -86 \\
\hline LJ-3641 & D7 & 540 & 1 & -24.9 & 4650 & 45 & 27 \\
\hline LJ -3659 & D7 & 550 & 1 & -24.5 & 4642 & 47 & -132 \\
\hline LJ J-3646 & D7 & 560 & 1 & -24.6 & 4673 & 45 & -55 \\
\hline LJ J-3657 & D7 & 570 & 1 & -24.8 & 4608 & 45 & 20 \\
\hline LJ -3642 & D7 & 580 & 1 & -24.5 & 4597 & 46 & -10 \\
\hline LJ-3666 & D7 & 590 & 1 & -24.4 & 4614 & 45 & 77 \\
\hline LJ J-3658 & D7 & 600 & 1 & -24.7 & 4576 & 46 & 54 \\
\hline LJ J-3007 & D7 & 608 & 3 & -28.5 & 4642 & 43 & 123 \\
\hline LJ J-2957 & D7 & 614 & 1 & -28.1 & 4564 & 42 & -58 \\
\hline LJ-2956 & D7 & 619 & 1 & -27.8 & 4612 & 42 & -9 \\
\hline LJ-3081 & D7 & 630 & 1 & -26.8 & 4604 & 44 & -26 \\
\hline$L J-3111$ & D7 & 636 & 1 & -26.8 & 4647 & 45 & -44 \\
\hline LJ J-3015 & D7 & 643 & 1 & -26.2 & 4715 & 52 & 42 \\
\hline LJ-3938 & D7 & 649 & 1 & -26.6 & 4651 & 46 & -15 \\
\hline LJ J-3116 & D7 & 651 & 1 & -25.8 & 4663 & 44 & -6 \\
\hline LJ-3931 & D7 & 656 & 1 & -26.7 & 4679 & 46 & 19 \\
\hline LJ-3017 & D7 & 670 & 1 & -26.0 & 4636 & 45 & 43 \\
\hline LJ J-3078 & $\mathrm{D} 7$ & 674 & 1 & -26.1 & 4627 & 45 & -48 \\
\hline LJ J-3018 & 107 & 680 & 1 & -24.5 & 4552 & 45 & 47 \\
\hline LJ-3928 & D7 & 689 & 1 & -26.4 & 4449 & 46 & 140 \\
\hline LJ -2976 & D7 & 693 & I & -26.0 & 4487 & 43 & -97 \\
\hline LJ J-3930 & D7 & 695 & 1 & -25.6 & 4528 & 46 & -108 \\
\hline LJ J-3009 & D7 & 698 & 1 & -26.0 & 4458 & 42 & 26 \\
\hline LJ -2975 & I) 7 & 712 & 1 & -26.2 & 4498 & 42 & -30 \\
\hline LJJ-3132 & $\mathrm{D} 7$ & 727 & 1 & -25.1 & 4479 & 44 & 116 \\
\hline LJ-3082 & D7 & 730 & 1 & -25.8 & 4405 & 44 & 2 \\
\hline LJ-3935 & D7 & 732 & 1 & -25.4 & 4494 & 46 & 83 \\
\hline LJ J-3131 & D7 & 734 & 1 & -25.2 & 4426 & 45 & -14 \\
\hline LJ-3936 & 107 & 751 & 1 & -25.9 & 4417 & 46 & -58 \\
\hline LJ J-3113 & D7 & 757 & 1 & -25.6 & 4473 & 43 & 84 \\
\hline LJ J-3933 & D7 & 760 & 1 & -25.8 & 4451 & 45 & 92 \\
\hline LJ J-2960 & D7 & 761 & 1 & -25.7 & 4469 & 42 & 21 \\
\hline LJ J-3016 & I)7 & 767 & 1 & -24.4 & 4417 & 44 & 45 \\
\hline LJ J-2958 & D7 & 773 & I & -24.9 & 4439 & 44 & -86 \\
\hline LJ J-2959 & D7 & 799 & 1 & $(-26.2)$ & 4441 & 42 & -90 \\
\hline
\end{tabular}

Main 5 series, B Becker, estimated 7ero point ca 3250 BC (uncertain)

$\begin{array}{llrllllr}\text { LJ-3698 } & \text { M5 } & 73 & 5 & -25.0 & 4418 & 45 & 59 \\ \text { LJ-3739 } & \text { M5 } & 126 & 2 & -26.2 & 4471 & 45 & 104 \\ \text { LJ-3591 } & \text { M5 } & 154 & 8 & -26.3 & 4475 & 47 & -88 \\ \text { LJ-3738 } & \text { M5 } & 200 & 1 & -25.0 & 4392 & 44 & -225 \\ \text { LJ-3740 } & \text { M5 } & 225 & 1 & -25.2 & 4369 & 45 & -121 \\ \text { LJ-3702 } & \text { M5 } & 256 & 1 & -23.8 & 4315 & 44 & -57 \\ \text { LJ-3742 } & \text { M5 } & 274 & 2 & -24.9 & 4397 & 45 & -1 \\ \text { LJ-3741 } & \text { M5 } & 305 & 1 & -25.8 & 4296 & 44 & 23 \\ \text { LJ-3701 } & \text { M5 } & 325 & 1 & -23.8 & 4335 & 44 & -59 \\ \text { LJ-3694 } & \text { M5 } & 350 & 1 & -24.1 & 4270 & 45 & 82 \\ \text { LJ-3737 } & \text { M5 } & 373 & 1 & -25.9 & 4219 & 44 & -81 \\ \text { LJ-3693 } & \text { M5 } & 399 & 1 & -23.2 & 4192 & 44 & 23 \\ \text { LJ-3743 } & \text { M5 } & 440 & 2 & -24.5 & 4080 & 44 & 75\end{array}$


Appendix 2 (continued)

\begin{tabular}{|c|c|c|c|c|c|c|c|}
\hline 1. & 2. & 3. & 4. & 5. & 6. & 7. & 8. \\
\hline \multicolumn{8}{|c|}{$\begin{array}{l}\text { Donau } 3 \text { and Donau } 10 \text { series, Bronze Age master chronology of } \\
\text { B Becker, estimated zero point ca } 2860 \mathrm{BC}\end{array}$} \\
\hline LJ -3458 & D3/10 Ner 51-61 & 45 & 2 & -24.9 & 4125 & 43 & 59 \\
\hline LJ -3696 & D3/10 Ner 51-61 & 74 & 1 & -23.9 & 4044 & 44 & -26 \\
\hline LJ -3440 & D3/10 Ner $51-61$ & 80 & 1 & -24.6 & 4118 & 43 & -62 \\
\hline LJ -3436 & D3/10 Ner $51-61$ & 130 & 1 & -24.7 & 4171 & 43 & 0 \\
\hline LJ -3691 & D3/10 Ner 51-61 & 145 & 1 & -25.7 & 4154 & 44 & 50 \\
\hline LJ -3692 & D3/10 Ner 51-61 & 171 & 1 & -26.3 & 4125 & 44 & -83 \\
\hline LJ J-3464 & I3/10 Ner 51-61 & 209 & 1 & -25.2 & 4088 & 60 & B \\
\hline LJ -3465 & D3/10 Ner 51-61 & 226 & 1 & -24.3 & 4072 & 61 & B \\
\hline LJ -3457 & 1)3/10 Ner 51-61 & 250 & 1 & -24.2 & 4041 & 43 & 6 \\
\hline LJ -3695 & D3/10 Ner 51-61 & 250 & 2 & -23.7 & 4040 & 44 & -65 \\
\hline LJ -3699 & D3/10 Ner $51-61$ & 261 & $\overrightarrow{1}$ & -24.2 & 4106 & 44 & -67 \\
\hline LJ -3437 & I) $3 / 10$ Ner $5 \mathrm{I}-6 \mathrm{I}$ & 270 & 1 & $-\overline{2} 4.3$ & 4076 & 43 & 75 \\
\hline LJ -3703 & D3/10 Ner $51-61$ & 280 & 2 & -24.0 & 3978 & 44 & -10 \\
\hline $\mathbf{L J}-3294$ & D3/10 Ner 51-61 & 301 & 1 & -24.2 & 3940 & 43 & 6 \\
\hline L.J -3700 & $1) 3 / 10$ Ner $51-61$ & 310 & 1 & -24.1 & 4117 & 44 & 55 \\
\hline LJ J -3290 & D3/10 Ner 51-61 & 328 & 1 & -24.4 & 3930 & 60 & $\mathbf{M}$ \\
\hline LJ -3291 & D3/10 Ner 51-61 & 343 & 1 & -25.0 & 3918 & 43 & -56 \\
\hline LJ -3704 & D3/10 Ner 47 & 388 & 1 & -26.0 & 3911 & 44 & -9 \\
\hline LJ -3706 & D3/10 Ner 36 & 400 & 1 & -23.9 & 3818 & 43 & -42 \\
\hline $\mathrm{LJ}-3705$ & D3/10 Ner 1 & 420 & 1 & -24.3 & 3824 & 43 & 64 \\
\hline $\mathbf{L J}-3776$ & D3 Bur H534 & 430 & 1 & -26.5 & 3788 & 81 & M \\
\hline $\mathbf{L J}-3774$ & D3 Bur H52 & 470 & 1 & $-2,5.7$ & 3791 & 43 & 31 \\
\hline LJ -3773 & D3 Вuг $\mathrm{H52}$ & 476 & 1 & -25.6 & 3823 & 62 & M \\
\hline LJ -3806 & D3 Bur H52 & 490 & 1 & --25.2 & 3992 & 42 & 202 \\
\hline LJ -3292 & $193 / 10$ Bur H26 & 500 & 1 & -24.7 & 3842 & 43 & -55 \\
\hline LJ -3779 & D3 Bur H52 & 515 & 1 & -25.4 & 3819 & 44 & 135 \\
\hline LJ -3793 & D3 Bur H52 & 540 & 1 & -24.6 & 3816 & 45 & 48 \\
\hline LJ -3293 & 1)3/10 Bur H26 & 542 & 1 & -25.2 & 3816 & 41 & -95 \\
\hline LJ J-3778 & D3 Bur H52 & 560 & 1 & -26.4 & 3754 & 48 & -10 \\
\hline LJ -3791 & 1)3 Ner 4 & 570 & 1 & -25.6 & 3801 & 60 & B \\
\hline LJ J -3792 & D3 Ner 4 & 575 & 1 & -24.0 & 3826 & 46 & -129 \\
\hline $\mathrm{LJ}-3813$ & D3 Ner 4 & 590 & 1 & -24.6 & 3793 & 46 & -140 \\
\hline LJ-3439 & 1)3/10 Bur H26 & 604 & 1 & -24.7 & 3855 & 43 & -96 \\
\hline LJ -3807 & I) 3 Lei 42 & 645 & 1 & -24.8 & 3699 & 45 & -78 \\
\hline LJ-3441 & D3/10 Lei 39 & 650 & 1 & -25.3 & 3790 & 42 & -84 \\
\hline LJ -3808 & D3 Lei 42 & 665 & 1 & -23.5 & 3715 & 60 & B \\
\hline LJ -3724 & D3/10 Gun 34 & 676 & 1 & -23.9 & 3685 & 43 & -3 \\
\hline $\mathbf{L J}-3782$ & D3 Lei 42 & 685 & 1 & -24.5 & 3664 & 45 & -32 \\
\hline LJ J-3462 & D3/10 Lei 39 & 700 & 1 & -24.5 & 3667 & 42 & 18 \\
\hline LJ-3780 & D3 Bur 15 & 710 & 1 & -25.4 & 3659 & 45 & 30 \\
\hline LJ-3721 & D3/10 Gun 34 & 725 & 1 & -23.8 & 3789 & 43 & -38 \\
\hline LJ -3775 & D3 Bur 15 & 736 & 1 & -26.4 & 3811 & 43 & -82 \\
\hline LJ -3781 & D3 Bur 15 & 745 & 1 & -24.9 & 3710 & 45 & 49 \\
\hline LJ -3463 & D3/10 Lei 39 & 750 & 1 & -24.7 & 3668 & 42 & -67 \\
\hline LJ -3789 & D3 Bur 15 & 750 & 1 & -24.3 & 3652 & 46 & -9 \\
\hline LJ-3788 & D3 Bur 15 & 765 & 1 & -24.8 & 3606 & 46 & 19 \\
\hline LJ J -3723 & D3/10 Gun 34 & 774 & 1 & -23.8 & 3641 & 43 & 49 \\
\hline LJ-3438 & 1)3/10 Lei 39 & 800 & 1 & -24.6 & 3716 & 42 & -108 \\
\hline L.J-3765 & $103 / 10$ Bur 15 & 817 & 1 & -26.0 & 3585 & 43 & 8 \\
\hline Lj-3725 & D3/10 Gun 34 & 832 & 1 & -24.5 & 3626 & 42 & -213 \\
\hline LJ -3722 & $193 / 10$ Gun 37 & 856 & 1 & -25.1 & 3566 & 42 & 1 \\
\hline LJ -3727 & 1) $3 / 10$ Gun 37 & 873 & 1 & -26.2 & 3631 & 43 & 8 \\
\hline LJ J 3759 & D3/10 Gun 37 & 897 & 1 & -26.5 & 3749 & 43 & 62 \\
\hline $\mathrm{LJ}-3764$ & D3/10 (;un 37 & 925 & 1 & -26.1 & 3516 & 42 & 35 \\
\hline $\mathrm{LJJ}-3726$ & 1) $3 / 10$ Gun 37 & 947 & 1 & -25.2 & 3520 & 42 & -53 \\
\hline LJ J -3766 & 1) $3 / 106$ Gi 2 & 975 & 1 & -23.9 & 3617 & 43 & 36 \\
\hline LJ -3785 & D3 Bur 1 & 985 & 1 & -24.9 & 3480 & 42 & -19 \\
\hline LJ -3783 & D3 Bur 1 & 995 & 1 & -25.3 & 3429 & 42 & -127 \\
\hline
\end{tabular}


Appendix 2 (continued)

\begin{tabular}{clccccrr}
\hline 1. & \multicolumn{1}{c}{2.} & 3. & 4. & 5. & 6. & 7. & 8. \\
\hline LJ-3757 & D3/10 Gri 2 & 1008 & 1 & -24.2 & 3484 & 42 & -15 \\
LJ-3763 & D3/10 Bur H & 1027 & 3 & -24.6 & 3500 & 42 & -225 \\
LJ-3814 & D3 Bur 1 & 1035 & 1 & -24.1 & 3444 & 45 & 9 \\
LJ-3443 & D3/10 Bur H22 & 1050 & 1 & -25.3 & 3458 & 42 & -64 \\
LJ-3811 & D3 Bur 1 & 1060 & 1 & -23.6 & 3385 & 45 & 117 \\
LJ-3767 & D3/10 Bur H22 1070 & 2 & -23.6 & 3451 & 42 & -41 \\
LJ-3784 & D3 Bur 1 & 1090 & 1 & -23.0 & 3471 & 42 & -30 \\
LJ-3459 & D3/10 Bur H22 & 1100 & 1 & -24.4 & 3485 & 42 & 33 \\
LJ-3760 & D3/10 Hoc 67 1125 & 1 & -25.0 & 3439 & 42 & 8 \\
LJ-3461 & D3/10 Bur H22 & 1150 & 1 & -25.3 & 3383 & 59 & M \\
LJ-3758 & D3/10 Hoc 67 1173 & 1 & -25.3 & 3417 & 42 & -32 \\
LJ-3460 & D3/10 Bur H22 & 1200 & 1 & -25.6 & 3435 & 42 & 48 \\
LJ-3442 & D3/10 Bur H22 & 1250 & 1 & -25.4 & 3338 & 41 & 123
\end{tabular}

Zug Sumpf series, obtained from Veronika Giertz Siebenlist, estimated zero point ca $1250 \mathrm{BC}$

\begin{tabular}{llrllllr} 
LJ-3028 & Giertz & 28 & 14 & -27.3 & 2915 & 39 & -36 \\
LJ-2917 & Giertz & 50 & 10 & -27.8 & 2899 & 39 & -46 \\
LJ-2949 & Giertz & 70 & 10 & -26.5 & 2877 & 39 & 17 \\
LJ-2918 & Giertz & 90 & 10 & -27.9 & 2892 & 39 & -40 \\
LJ-2916 & Giertz & 110 & 10 & -27.6 & 2951 & 39 & 20 \\
LJ-2920 & Giertz & 130 & 10 & -26.4 & 2815 & 39 & -28 \\
LJ-2919 & Giertz & 150 & 10 & -26.4 & 2885 & 39 & 89 \\
LJ-2915 & Giertz & 170 & 10 & -28.6 & 2762 & 39 & -70 \\
LJ-2939 & Giertz & 190 & 10 & -26.7 & 2842 & 39 & 78 \\
LJ-2922 & Giertz & 210 & 10 & -25.2 & 2796 & 39 & 56 \\
LJ-3024 & Giertz & 245 & 10 & -26.1 & 2787 & 38 & -8 \\
LJ-2921 & Giertz & 260 & 10 & -26.6 & 2709 & 39 & 117 \\
LJ-3025 & Giertz & 280 & 10 & -27.8 & 2723 & 40 & -17 \\
\hline
\end{tabular}

RFFHRNCES

Becker, B, 1978, Holocene tree-ring series from southern Central Europe for archaeologic dating, radiocarbon calibration, and stable isotope analysis: Internat. Radiocarbon Conference, IX, Los Angeles and Ia Jolla, June 1976, Proc., in press.

Cain, W F, and Suess, H E, 1976, Carbon-14 in tree rings: Jour Geophys Rescarch, v 81, p $3688-3694$

Ferguson, C W, 1968, Bristlecone pine: science and esthetics: Science, v 159, p 839-846.

Ferguson, C W, Huber, B, and Suess, H E, 1966, Determination of age of Swiss lake dwellings as an example of dendrochronologically-calibrated radiocarbon dating: Zeitsch Naturforschg, v $21 \mathrm{a}, \mathrm{p} 1173-1177$.

Linick, T W, 1977, La Jolla Natural Radiocarbon Measurements VII: Radiocarbon, v 19 , no 1, p $19-48$.

Stuiver, M, and Suess, H E, 1966, On the relationship between radiocarbon dates and true sample ages: Radiocarbon, v 8, p 534-540.

Suess, H E, 1965, Secular variations in the cosmic ray-produced carbon-14 in the atmosphere and their interpretations: Jour Geophys Research, v 70, p 5937-5952. 1971, Bristlecone pine calibration of the radiocarbon time scale 5200 B.C. to the present, in Olsson, I, ed, Nobel Symposium Radiocarbon Variations and Abs Chronology, Uppsala 1969: p 303-313. 1978, The Carbon-14 level during the Fourth and Sccond Half of the Fifth Millenium BC and the Carbon-14 Calibration Curve: IX Internat. Radiocarbon Conference, 9th, Los Angeles and La Jolla, June 1976, Proc., in press.

Suess, H E, and Becker, B, 1977, Der Radiokarbongehalt von Jahresringproben aus postglazialen Eichenstämmen Südmitteleuropas, Erdwissenschaftliche Forschung, v 13, Dendrochronologie and postglaziale Klimaschwankungen: Wiesbaden, Franz Steiner Verlag, p 15-170. 